\title{
Rhodium-Catalyzed Interconversion of Quinolinyl Ketones with Boronic Acids via C-C Bond Activation
}

\author{
Joseph M. Dennis, Chad T. Compagner, \\ Stanna K. Dorn, Jeffrey B. Johnson* \\ Department of Chemistry, Hope College, Holland, MI 49423 \\ jjohnson@hope.edu
}

Supporting Information

\begin{tabular}{ll}
\multicolumn{1}{c}{ Index } \\
General Methods and Procedures & S2 \\
Optimization and Substrate Scope & S4 \\
Characterization Data & S9 \\
NMR Spectra & S17 \\
References & S33
\end{tabular}


General Methods. All reactions were carried out under an atmosphere of nitrogen or argon in oven-dried glassware with magnetic stirring. Solvents, including toluene, tetrahydrofuran (THF), dimethylformamide (DMF) and dimethyl sulfoxide (DMSO), were purged with argon and passed through two columns of neutral alumina or molecular sieves. Quinolinyl ketones 1, 2, 4, 14 and 15 were prepared according to literature precedent. ${ }^{1,2,3}$ Starting materials were obtained from Alfa Aesar or Aldrich Chemical and used with further purification. Catalysts, specifically $\left[\mathrm{Rh}\left(\mathrm{C}_{2} \mathrm{H}_{4}\right)_{2} \mathrm{Cl}\right]_{2}$ and $\mathrm{Rh}\left(\mathrm{PPh}_{3}\right)_{3} \mathrm{Cl}$, were obtained from Strem Chemical, Inc. or Pressure Chemical Co. and utilized without further purification. ${ }^{1} \mathrm{H}$ and ${ }^{13} \mathrm{C}$ NMR spectra were obtained on a Bruker Avance III $400 \mathrm{MHz}$ spectrometer using standard acquisition parameters.

Method for Synthesis of Quinolinyl Ketones will be illustrated with a specific example. A oven-dried $250 \mathrm{~mL}$ round bottomed flask was charged with 8bromoquinoline ( $2.81 \mathrm{~g}, 13.5 \mathrm{mmol}$ ) and dissolved in THF under an Ar atmosphere. The reaction was cooled to $-78^{\circ} \mathrm{C}$ and $n \mathrm{BuLi}(2.5 \mathrm{M}$ in hexanes, $6 \mathrm{~mL}, 15.0 \mathrm{mmol})$ was added over approximately 15 minutes. 2-Fluorobenzaldehdye $(1.58 \mathrm{~mL}, 15$ mmol) was added via syringe over approximately 10 minutes. Following addition, the reaction was allowed to warm to room temperature and stirred for 2 hours. The reaction mixture was quenched with sat. $\mathrm{NH}_{4} \mathrm{Cl}(50 \mathrm{~mL})$ and the layers separated. The organic layer was washed with water $(20 \mathrm{~mL})$, and the combined aqueous layers were extracted with ether $(2 \times 50 \mathrm{~mL})$, dried over $\mathrm{Na}_{2} \mathrm{SO}_{4}$ and concentrated under reduced pressure. The resulting residue was dissolved in DMSO $(40 \mathrm{~mL})$ and IBX ( $4.38 \mathrm{~g}, 15.0 \mathrm{mmol}$ ) was added. The solution was allowed to stir at room temperature for 2 hours. A solution of 1:1 EtOAc: $\mathrm{H}_{2} \mathrm{O}(50 \mathrm{~mL})$ was added and the reaction mixture was filtered through a pad of Celite. The layers were separated and the aqueous layer was extracted with EtOAc $(3 \times 10 \mathrm{~mL})$. The combined organic layers were washed with brine, dried over $\mathrm{Na}_{2} \mathrm{SO}_{4}$ and concentrated under reduced pressure. The orange oil was purified by column chromatography (9:1 hexane:EtOAc) to provide the product as an orange oil. 


\section{Method A: Ketone Exchange with Aryl Quinolinyl Ketones and Aryl Boronic}

Acids will be illustrated with a specific example. An oven-dried $5 \mathrm{~mL}$ pressure tube with a stir bar was charged with phenyl boronic acid (34.1 mg, $0.28 \mathrm{mmol}$ ) and 2methoxyquinolinyl ketone 4 (30.0 $\mathrm{mg}, 0.11 \mathrm{mmol}$ ) and transferred into an inert atmosphere glovebox. To the tube was added $\left[\mathrm{Rh}\left(\mathrm{C}_{2} \mathrm{H}_{4}\right)_{2} \mathrm{Cl}\right]_{2}(2.5 \mathrm{mg}, 0.006 \mathrm{mmol})$ and $2 \mathrm{~mL}$ of toluene. The tube was sealed with a Teflon cap, removed from the glovebox and placed in a $140^{\circ} \mathrm{C}$ oil bath for $48 \mathrm{~h}$. After cooling to room temperature, the reaction mixture was filtered through a short silica gel plug with additional ethyl acetate. The organic solution was washed with $2 \mathrm{M} \mathrm{NaOH}$ and the aqueous layer was extracted with ethyl acetate $(3 \times 10 \mathrm{~mL})$. The combined organic layers were washed with brine, dried over $\mathrm{Na}_{2} \mathrm{SO}_{4}$ and concentrated under reduced pressure. Column chromatography (9:1 hexane:EtOAc) yielded 2 as an orange solid (14 mg, 0.06 mmol, $52 \%$ yield).

\section{Method B: Ketone Exchange with Phenethyl Quinolinyl Ketone 15 and Aryl}

Boronic Acids will be illustrated with a specific example. An oven-dried $5 \mathrm{~mL}$ pressure tube with a stirbar was charged with phenylboronic acid $(29.0 \mathrm{mg}, 0.24$ $\mathrm{mmol})$ and phenethyl quinolinyl ketone $15(25.0 \mathrm{mg}, 0.10 \mathrm{mmol})$ and transferred into an inert atmosphere glovebox. To the tube was added $\mathrm{Rh}\left(\mathrm{PPh}_{3}\right)_{3} \mathrm{Cl}(8.9 \mathrm{mg}$, $0.010 \mathrm{mmol}$ ) and $2 \mathrm{~mL}$ of toluene. The tube was sealed with a Teflon cap, removed from the glovebox and placed in a $140{ }^{\circ} \mathrm{C}$ oil bath for $48 \mathrm{~h}$. After cooling to room temperature, the reaction mixture was filtered through a short silica gel plug with additional ethyl acetate. The organic solution was washed with $2 \mathrm{M} \mathrm{NaOH}$ and the aqueous layer was extracted with ethyl acetate $(3 \times 10 \mathrm{~mL})$. The combined organic layers were washed with brine $(20 \mathrm{~mL})$, dried over $\mathrm{Na}_{2} \mathrm{SO}_{4}$ and concentrated under reduced pressure. Column chromatography (9:1 hexane:EtOAc) yielded 2 as a white solid (14 $\mathrm{mg}, 0.06 \mathrm{mmol}, 63 \%$ yield).

Method C: Ketone Exchange with Methyl Boronic Acid will be illustrated with a specific example. An oven-dried $5 \mathrm{~mL}$ pressure tube with a stir bar was charged with $\mathrm{MeB}(\mathrm{OH})_{2}(19.5 \mathrm{mg}, 0.325 \mathrm{mmol}), \mathrm{K}_{2} \mathrm{CO}_{3}$ ( $48.5 \mathrm{mg}, 0.350 \mathrm{mmol}$ ) and phenyl 
quinolinyl ketone 2 (30 $\mathrm{mg}, 0.128 \mathrm{mmol}$ ) and transferred into an inert atmosphere glovebox. To the tube was added $\left[\mathrm{Rh}\left(\mathrm{C}_{2} \mathrm{H}_{4}\right)_{2} \mathrm{Cl}\right]_{2}(2.6 \mathrm{mg}, 0.0067 \mathrm{mmol})$ and $2 \mathrm{~mL}$ of toluene. The tube was sealed with a Teflon cap, removed from the glovebox and placed in a $140^{\circ} \mathrm{C}$ oil bath for $48 \mathrm{~h}$. After cooling to room temperature, the reaction mixture was filtered through a short silica gel plug with additional ethyl acetate. The organic solution was washed with $2 \mathrm{M} \mathrm{NaOH}$ and the aqueous layer was extracted with ethyl acetate. The combined organic layers were washed with brine, dried over $\mathrm{Na}_{2} \mathrm{SO}_{4}$ and concentrated under reduced pressure. Column chromatography (9:1 hexane:EtOAc) yielded the desired product as a cream colored solid.

\section{Table S1. Catalyst testing}

[Rh], $\mathrm{R} ' \mathrm{~B}(\mathrm{OH})_{2}$
Entry $^{\boldsymbol{a}}$


Table S2. Additional reaction optimization

\begin{tabular}{|c|c|c|c|c|c|}
\hline Entry $^{a}$ & Solvent & Equiv $\mathrm{RB}(\mathrm{OH})_{2}$ & Temp $\left({ }^{\circ} \mathrm{C}\right)$ & Catalyst & Yield (\%) \\
\hline 1 & Toluene & 2.5 & 140 & $\mathrm{Rh}\left(\mathrm{PPh}_{3}\right)_{3} \mathrm{Cl}$ & 42 \\
\hline 2 & Toluene & 2.5 & 140 & {$\left[\mathrm{Rh}\left(\mathrm{C}_{2} \mathrm{H}_{4}\right)_{2} \mathrm{Cl}\right]_{2}$} & 55 \\
\hline 3 & $\mathrm{THF}$ & 2.5 & 90 & {$\left[\mathrm{Rh}\left(\mathrm{C}_{2} \mathrm{H}_{4}\right)_{2} \mathrm{Cl}\right]_{2}$} & $<10$ \\
\hline 4 & $o$-Xylene & 2.5 & 140 & {$\left[\mathrm{Rh}\left(\mathrm{C}_{2} \mathrm{H}_{4}\right)_{2} \mathrm{Cl}\right]_{2}$} & 32 \\
\hline 5 & $o$-Xylene & 2.5 & 140 & $\mathrm{Rh}\left(\mathrm{PPh}_{3}\right)_{3} \mathrm{Cl}$ & 20 \\
\hline 6 & 1,4-dioxane & 2.5 & 110 & {$\left[\mathrm{Rh}\left(\mathrm{C}_{2} \mathrm{H}_{4}\right)_{2} \mathrm{Cl}\right]_{2}$} & $<10$ \\
\hline 7 & Toluene & 1 & 140 & $\mathrm{Rh}\left(\mathrm{PPh}_{3}\right)_{3} \mathrm{Cl}$ & 36 \\
\hline 8 & Toluene & 5 & 140 & $\mathrm{Rh}\left(\mathrm{PPh}_{3}\right)_{3} \mathrm{Cl}$ & 43 \\
\hline 9 & Toluene & 1 & 140 & {$\left[\mathrm{Rh}\left(\mathrm{C}_{2} \mathrm{H}_{4}\right)_{2} \mathrm{Cl}\right]_{2}$} & 42 \\
\hline
\end{tabular}

Table S3. Examination of Alternative Nucleophiles<smiles>[R]C(=O)c1cccc2cccnc12</smiles>

\begin{tabular}{llllll}
\hline Entry $^{\boldsymbol{a}}$ & $\mathbf{R}$ & Nucleophile & R' $^{\prime}$ & Catalyst & Yield (\%) \\
\hline 1 & $2-\mathrm{OMeC}_{6} \mathrm{H}_{4}$ & $\mathrm{PhB}(\mathrm{OH})_{2}$ & $\mathrm{Ph}$ & {$\left[\mathrm{Rh}\left(\mathrm{C}_{2} \mathrm{H}_{4}\right)_{2} \mathrm{Cl}\right]_{2}$} & 67 \\
2 & $2-\mathrm{OMeC}_{6} \mathrm{H}_{4}$ & $\mathrm{PhBpin}$ & $\mathrm{Ph}$ & $\mathrm{Rh}\left(\mathrm{PPh}_{3}\right)_{3} \mathrm{Cl}$ & $<5$ \\
3 & $2-\mathrm{OMeC}_{6} \mathrm{H}_{4}$ & $\mathrm{PhBpin}$ & $\mathrm{Ph}$ & {$\left[\mathrm{Rh}\left(\mathrm{C}_{2} \mathrm{H}_{4}\right)_{2} \mathrm{Cl}\right]_{2}$} & $<10$ \\
4 & $2-\mathrm{OMeC}_{6} \mathrm{H}_{4}$ & $\mathrm{BnBpin}$ & $\mathrm{Bn}$ & $\mathrm{Rh}\left(\mathrm{PPh}_{3}\right)_{3} \mathrm{Cl}$ & $<5$ \\
5 & $2-\mathrm{OMeC}_{6} \mathrm{H}_{4}$ & $\mathrm{Ph}_{2} \mathrm{Zn}$ & $\mathrm{Ph}$ & $\mathrm{Rh}\left(\mathrm{PPh}_{3}\right)_{3} \mathrm{Cl}$ & $<10$ \\
6 & $2-\mathrm{OMeC}_{6} \mathrm{H}_{4}$ & $\mathrm{Bu}_{3} \mathrm{Sn}-a l l y l$ & $\mathrm{CH}_{2} \mathrm{CH}=\mathrm{CH}_{2}$ & {$\left[\mathrm{Rh}\left(\mathrm{C}_{2} \mathrm{H}_{4}\right)_{2} \mathrm{Cl}\right]_{2}$} & $<5$ \\
$7^{c}$ & $\mathrm{Ph}$ & $\mathrm{MeBF}_{3} \mathrm{~K}$ & $\mathrm{Me}$ & {$\left[\mathrm{Rh}\left(\mathrm{C}_{2} \mathrm{H}_{4}\right)_{2} \mathrm{Cl}\right]_{2}$} & $<5$ \\
8 & $\mathrm{Ph}$ & $\mathrm{MeBF}_{3} \mathrm{~K}$ & $\mathrm{Me}$ & {$\left[\mathrm{Rh}\left(\mathrm{C}_{2} \mathrm{H}_{4}\right)_{2} \mathrm{Cl}\right]_{2}$} & $<5$ \\
\hline
\end{tabular}

$a$ Reaction conditions: Phenyl quinolinyl ketone $(0.11 \mathrm{mmol})$, nucleophile $(0.27 \mathrm{mmol})$, catalyst $(0.01 \mathrm{mmol}$ in $\mathrm{Rh})$, toluene $(2 \mathrm{~mL}), 140^{\circ} \mathrm{C}$ for 48 hours. $b$ Product formation based on conversion of starting materials by $\mathrm{GC} / \mathrm{MS}$ analysis. $c$ Reaction performed with $0.27 \mathrm{mmol} \mathrm{K}_{2} \mathrm{CO}_{3}$. 
Table S4. Reactions with Phenyl Quinolinyl Ketone

\author{
Catalyst

\begin{tabular}{llll}
\hline Entry $^{a}$ & R' & Catalyst & Yield \\
\hline 1 & $4-\mathrm{MeC}_{6} \mathrm{H}_{4}$ & {$\left[\mathrm{Rh}\left(\mathrm{C}_{2} \mathrm{H}_{4}\right)_{2} \mathrm{Cl}_{2}\right.$} & 55
\end{tabular} \\ $2 \quad 3,5-\left(\mathrm{CF}_{3}\right)_{2} \mathrm{C}_{6} \mathrm{H}_{3} \quad\left[\mathrm{Rh}\left(\mathrm{C}_{2} \mathrm{H}_{4}\right)_{2} \mathrm{Cl}\right]_{2} \quad 35$ \\ $3 \quad 4-\mathrm{C}(\mathrm{O}) \mathrm{CH}_{3} \quad\left[\mathrm{Rh}\left(\mathrm{C}_{2} \mathrm{H}_{4}\right)_{2} \mathrm{Cl}\right]_{2}<10$ \\ $4 \quad 3-\mathrm{C}(\mathrm{O}) \mathrm{CH}_{3} \quad\left[\mathrm{Rh}\left(\mathrm{C}_{2} \mathrm{H}_{4}\right)_{2} \mathrm{Cl}\right]_{2}<10$ \\ $5 \quad 3-\mathrm{CF}_{3} \quad\left[\mathrm{Rh}\left(\mathrm{C}_{2} \mathrm{H}_{4}\right)_{2} \mathrm{Cl}\right]_{2} \quad 53$ \\ $6 \quad 4-\mathrm{MeOC}_{6} \mathrm{H}_{4} \quad\left[\mathrm{Rh}\left(\mathrm{C}_{2} \mathrm{H}_{4}\right)_{2} \mathrm{Cl}\right]_{2} \quad<5$ \\ $7 \quad 4-\mathrm{CF}_{3} \quad\left[\mathrm{Rh}\left(\mathrm{C}_{2} \mathrm{H}_{4}\right)_{2} \mathrm{Cl}\right]_{2} \quad 62$ \\ $8 \quad(E)-\mathrm{CH}=\mathrm{CHPh} \quad\left[\mathrm{Rh}\left(\mathrm{C}_{2} \mathrm{H}_{4}\right)_{2} \mathrm{Cl}\right]_{2}<5$ \\ $9 \quad 4-\mathrm{NMe}_{2} \mathrm{C}_{6} \mathrm{H}_{4} \quad\left[\mathrm{Rh}\left(\mathrm{C}_{2} \mathrm{H}_{4}\right)_{2} \mathrm{Cl}\right]_{2}<5$ \\ $10 \quad\left(\mathrm{CH}_{2}\right)_{2} \mathrm{Ph} \quad\left[\mathrm{Rh}\left(\mathrm{C}_{2} \mathrm{H}_{4}\right)_{2} \mathrm{Cl}\right]_{2}<10$ \\ $a$ Reaction conditions: Phenyl quinolinyl ketone $2(0.11 \mathrm{mmol})$, boronic \\ acid $(0.27 \mathrm{mmol}),\left[\mathrm{Rh}\left(\mathrm{C}_{2} \mathrm{H}_{4}\right)_{2} \mathrm{Cl}\right]_{2}(0.006 \mathrm{mmol})$, toluene $(2 \mathrm{~mL}), \mathrm{N}_{2}, 140{ }^{\circ} \mathrm{C}$, \\ $48 \mathrm{~h} . b$ Product formation based on conversion of starting materials by \\ $\mathrm{GC} / \mathrm{MS}$ analysis.
}


Table S5. Reactions with 2-Methoxyphenyl Quinolinyl Ketone

\begin{tabular}{|c|c|c|c|c|}
\hline Entry $^{a}$ & $\mathbf{R}$ & $\mathbf{R}^{\prime}$ & Catalyst & Yield \\
\hline 1 & $2-\mathrm{MeOC}_{6} \mathrm{H}_{4}$ & $3,5-\left(\mathrm{CF}_{3}\right)_{2} \mathrm{C}_{6} \mathrm{H}_{3}$ & $\mathrm{Rh}\left(\mathrm{PPh}_{3}\right)_{3} \mathrm{Cl}$ & 37 \\
\hline 2 & $2-\mathrm{MeOC}_{6} \mathrm{H}_{4}$ & $3-\mathrm{NO}_{2} \mathrm{C}_{6} \mathrm{H}_{4}$ & $\mathrm{Rh}\left(\mathrm{PPh}_{3}\right)_{3} \mathrm{Cl}$ & 60 \\
\hline 3 & $2-\mathrm{MeOC}_{6} \mathrm{H}_{4}$ & $4-\mathrm{CNC}_{6} \mathrm{H}_{4}$ & $\mathrm{Rh}\left(\mathrm{PPh}_{3}\right)_{3} \mathrm{Cl}$ & 77 \\
\hline 4 & $2-\mathrm{MeOC}_{6} \mathrm{H}_{4}$ & $4-\mathrm{CF}_{3} \mathrm{C}_{6} \mathrm{H}_{4}$ & $\mathrm{Rh}\left(\mathrm{PPh}_{3}\right)_{3} \mathrm{Cl}$ & 93 \\
\hline 5 & $2-\mathrm{MeOC}_{6} \mathrm{H}_{4}$ & 2-naphtyl & $\mathrm{Rh}\left(\mathrm{PPh}_{3}\right)_{3} \mathrm{Cl}$ & 76 \\
\hline 6 & $2-\mathrm{MeOC}_{6} \mathrm{H}_{4}$ & 1-naphtyl & $\mathrm{Rh}\left(\mathrm{PPh}_{3}\right)_{3} \mathrm{Cl}$ & $<5$ \\
\hline 7 & $2-\mathrm{MeOC}_{6} \mathrm{H}_{4}$ & $3,5-\left(\mathrm{CF}_{3}\right)_{2} \mathrm{C}_{6} \mathrm{H}_{3}$ & {$\left[\mathrm{Rh}\left(\mathrm{C}_{2} \mathrm{H}_{4}\right)_{2} \mathrm{Cl}\right]_{2}$} & 95 \\
\hline 8 & $2-\mathrm{MeOC}_{6} \mathrm{H}_{4}$ & $4-\mathrm{CNC}_{6} \mathrm{H}_{4}$ & {$\left[\mathrm{Rh}\left(\mathrm{C}_{2} \mathrm{H}_{4}\right)_{2} \mathrm{Cl}\right]_{2}$} & 84 \\
\hline 9 & $2-\mathrm{MeOC}_{6} \mathrm{H}_{4}$ & $4-\mathrm{CF}_{3} \mathrm{C}_{6} \mathrm{H}_{4}$ & {$\left[\mathrm{Rh}\left(\mathrm{C}_{2} \mathrm{H}_{4}\right)_{2} \mathrm{Cl}\right]_{2}$} & 98 \\
\hline 10 & $2-\mathrm{MeOC}_{6} \mathrm{H}_{4}$ & $4-\mathrm{CO}_{2} \mathrm{MeC}_{6} \mathrm{H}_{4}$ & {$\left[\mathrm{Rh}\left(\mathrm{C}_{2} \mathrm{H}_{4}\right)_{2} \mathrm{Cl}\right]_{2}$} & 95 \\
\hline 11 & $2-\mathrm{MeOC}_{6} \mathrm{H}_{4}$ & $4-\mathrm{C}(\mathrm{O}) \mathrm{MeC}_{6} \mathrm{H}_{4}$ & {$\left[\mathrm{Rh}\left(\mathrm{C}_{2} \mathrm{H}_{4}\right)_{2} \mathrm{Cl}\right]_{2}$} & 80 \\
\hline 12 & $2-\mathrm{MeOC}_{6} \mathrm{H}_{4}$ & $3-\mathrm{NO}_{2} \mathrm{C}_{6} \mathrm{H}_{4}$ & {$\left[\mathrm{Rh}\left(\mathrm{C}_{2} \mathrm{H}_{4}\right)_{2} \mathrm{Cl}\right]_{2}$} & 74 \\
\hline 13 & $2-\mathrm{MeOC}_{6} \mathrm{H}_{4}$ & $3-\mathrm{CF}_{3} \mathrm{C}_{6} \mathrm{H}_{4}$ & {$\left[\mathrm{Rh}\left(\mathrm{C}_{2} \mathrm{H}_{4}\right)_{2} \mathrm{Cl}\right]_{2}$} & 77 \\
\hline 14 & $2-\mathrm{MeOC}_{6} \mathrm{H}_{4}$ & $3-\mathrm{C}(\mathrm{O}) \mathrm{MeC}_{6} \mathrm{H}_{4}$ & {$\left[\mathrm{Rh}\left(\mathrm{C}_{2} \mathrm{H}_{4}\right)_{2} \mathrm{Cl}\right]_{2}$} & 85 \\
\hline 15 & $2-\mathrm{MeOC}_{6} \mathrm{H}_{4}$ & 2-napthyl & {$\left[\mathrm{Rh}\left(\mathrm{C}_{2} \mathrm{H}_{4}\right)_{2} \mathrm{Cl}\right]_{2}$} & 88 \\
\hline 16 & $2-\mathrm{MeOC}_{6} \mathrm{H}_{4}$ & $\mathrm{Ph}$ & {$\left[\mathrm{Rh}\left(\mathrm{C}_{2} \mathrm{H}_{4}\right)_{2} \mathrm{Cl}\right]_{2}$} & 67 \\
\hline 17 & $2-\mathrm{MeOC}_{6} \mathrm{H}_{4}$ & $\mathrm{Cy}$ & {$\left[\mathrm{Rh}\left(\mathrm{C}_{2} \mathrm{H}_{4}\right)_{2} \mathrm{Cl}\right]_{2}$} & $<5$ \\
\hline 18 & $2-\mathrm{MeOC}_{6} \mathrm{H}_{4}$ & 4-C(O) $\mathrm{NMe}_{2} \mathrm{C}_{6} \mathrm{H}_{4}$ & {$\left[\mathrm{Rh}\left(\mathrm{C}_{2} \mathrm{H}_{4}\right)_{2} \mathrm{Cl}\right]_{2}$} & $<5$ \\
\hline
\end{tabular}

Table S6. Reactions with Other Quinolinyl Ketones and Boronic Acids

\begin{tabular}{|c|c|c|c|c|}
\hline Entry $^{a}$ & $\mathbf{R}$ & $\mathbf{R}^{\prime}$ & Catalyst & Yield \\
\hline 1 & $2-\mathrm{FC}_{6} \mathrm{H}_{4}$ & $\mathrm{Ph}$ & $\mathrm{Rh}\left(\mathrm{PPh}_{3}\right)_{3} \mathrm{Cl}$ & 93 \\
\hline 2 & $4-\mathrm{FC}_{6} \mathrm{H}_{4}$ & $\mathrm{Ph}$ & $\mathrm{Rh}\left(\mathrm{PPh}_{3}\right)_{3} \mathrm{Cl}$ & 10 \\
\hline 3 & $\mathrm{Me}$ & $\mathrm{Ph}$ & $\mathrm{Rh}\left(\mathrm{PPh}_{3}\right)_{3} \mathrm{Cl}$ & 60 \\
\hline 4 & $4-\mathrm{CF}_{3} \mathrm{C}_{6} \mathrm{H}_{4}$ & $\mathrm{Ph}$ & $\mathrm{Rh}\left(\mathrm{PPh}_{3}\right)_{3} \mathrm{Cl}$ & $<5$ \\
\hline 5 & $2-\mathrm{FC}_{6} \mathrm{H}_{4}$ & 4- $\mathrm{MeC}_{6} \mathrm{H}_{4}$ & {$\left[\mathrm{Rh}\left(\mathrm{C}_{2} \mathrm{H}_{4}\right)_{2} \mathrm{Cl}\right]_{2}$} & $<5$ \\
\hline 6 & $2-\mathrm{FC}_{6} \mathrm{H}_{4}$ & 4- $\mathrm{MeC}_{6} \mathrm{H}_{4}$ & $\mathrm{Rh}\left(\mathrm{PPh}_{3}\right)_{3} \mathrm{Cl}$ & 87 \\
\hline 7 & $4-\mathrm{FC}_{6} \mathrm{H}_{4}$ & 4- $\mathrm{MeC}_{6} \mathrm{H}_{4}$ & {$\left[\mathrm{Rh}\left(\mathrm{C}_{2} \mathrm{H}_{4}\right)_{2} \mathrm{Cl}\right]_{2}$} & $<5$ \\
\hline 8 & $2-\mathrm{MeC}_{6} \mathrm{H}_{4}$ & $4-\mathrm{CF}_{3} \mathrm{C}_{6} \mathrm{H}_{4}$ & $\mathrm{Rh}\left(\mathrm{PPh}_{3}\right)_{3} \mathrm{Cl}$ & $<10$ \\
\hline 9 & $2-\mathrm{CF}_{3} \mathrm{C}_{6} \mathrm{H}_{4}$ & $4-\mathrm{CF}_{3} \mathrm{C}_{6} \mathrm{H}_{4}$ & {$\left[\mathrm{Rh}\left(\mathrm{C}_{2} \mathrm{H}_{4}\right)_{2} \mathrm{Cl}\right]_{2}$} & $<10$ \\
\hline
\end{tabular}


Table S7. Reactions with Phenethyl Quinolinyl Ketone

\begin{tabular}{|c|c|c|c|c|}
\hline Entry $^{a}$ & $\mathbf{R}$ & $\mathbf{R}^{\prime}$ & Catalyst & Yield \\
\hline 1 & $\mathrm{CH}_{2} \mathrm{CH}_{2} \mathrm{Ph}$ & $\mathrm{Ph}$ & $\mathrm{Rh}\left(\mathrm{PPh}_{3}\right)_{3} \mathrm{Cl}$ & 63 \\
\hline 2 & $\mathrm{CH}_{2} \mathrm{CH}_{2} \mathrm{Ph}$ & $4-\mathrm{CNC}_{6} \mathrm{H}_{4}$ & $\mathrm{Rh}\left(\mathrm{PPh}_{3}\right)_{3} \mathrm{Cl}$ & 92 \\
\hline 3 & $\mathrm{CH}_{2} \mathrm{CH}_{2} \mathrm{Ph}$ & $\mathrm{CH}_{3}$ & $\mathrm{Rh}\left(\mathrm{PPh}_{3}\right)_{3} \mathrm{Cl}$ & $<5$ \\
\hline 4 & $\mathrm{CH}_{2} \mathrm{CH}_{2} \mathrm{Ph}$ & $4-\mathrm{NMe}_{2} \mathrm{C}_{6} \mathrm{H}_{4}$ & $\mathrm{Rh}\left(\mathrm{PPh}_{3}\right)_{3} \mathrm{Cl}$ & $<5$ \\
\hline 5 & $\mathrm{CH}_{2} \mathrm{CH}_{2} \mathrm{Ph}$ & $4-\mathrm{CF}_{3} \mathrm{C}_{6} \mathrm{H}_{4}$ & $\mathrm{Rh}\left(\mathrm{PPh}_{3}\right)_{3} \mathrm{Cl}$ & 75 \\
\hline 6 & $\mathrm{CH}_{2} \mathrm{CH}_{2} \mathrm{Ph}$ & Hexyl & $\mathrm{Rh}\left(\mathrm{PPh}_{3}\right)_{3} \mathrm{Cl}$ & $<5$ \\
\hline 7 & $\mathrm{CH}_{2} \mathrm{CH}_{2} \mathrm{Ph}$ & $(E)-\mathrm{CH}=\mathrm{CHPh}$ & $\mathrm{Rh}\left(\mathrm{PPh}_{3}\right)_{3} \mathrm{Cl}$ & $<5$ \\
\hline 8 & $\mathrm{CH}_{2} \mathrm{CH}_{2} \mathrm{Ph}$ & 2- $\mathrm{CH}_{3} \mathrm{C}_{6} \mathrm{H}_{4}$ & $\mathrm{Rh}\left(\mathrm{PPh}_{3}\right)_{3} \mathrm{Cl}$ & 10 \\
\hline 9 & $\mathrm{CH}_{2} \mathrm{CH}_{2} \mathrm{Ph}$ & 2-napthyl & $\mathrm{Rh}\left(\mathrm{PPh}_{3}\right)_{3} \mathrm{Cl}$ & 45 \\
\hline 10 & $\mathrm{CH}_{2} \mathrm{CH}_{2} \mathrm{Ph}$ & $4-\mathrm{OMeC}_{6} \mathrm{H}_{4}$ & $\mathrm{Rh}\left(\mathrm{PPh}_{3}\right)_{3} \mathrm{Cl}$ & 33 \\
\hline 11 & $\mathrm{CH}_{2} \mathrm{CH}_{2} \mathrm{Ph}$ & $4-\mathrm{SMeC}_{6} \mathrm{H}_{4}$ & $\mathrm{Rh}\left(\mathrm{PPh}_{3}\right)_{3} \mathrm{Cl}$ & 41 \\
\hline 12 & $\mathrm{CH}_{2} \mathrm{CH}_{2} \mathrm{Ph}$ & $3-\mathrm{OMeC}_{6} \mathrm{H}_{4}$ & $\mathrm{Rh}\left(\mathrm{PPh}_{3}\right)_{3} \mathrm{Cl}$ & 52 \\
\hline 13 & $\mathrm{CH}_{2} \mathrm{CH}_{2} \mathrm{Ph}$ & $2-\mathrm{OMeC}_{6} \mathrm{H}_{4}$ & $\mathrm{Rh}\left(\mathrm{PPh}_{3}\right)_{3} \mathrm{Cl}$ & 5 \\
\hline 14 & $\mathrm{CH}_{2} \mathrm{CH}_{2} \mathrm{Ph}$ & $3,5-\left(\mathrm{CF}_{3}\right)_{2} \mathrm{C}_{6} \mathrm{H}_{3}$ & $\mathrm{Rh}\left(\mathrm{PPh}_{3}\right)_{3} \mathrm{Cl}$ & 52 \\
\hline 15 & $\mathrm{CH}_{2} \mathrm{CH}_{2} \mathrm{Ph}$ & $4-\mathrm{CO}_{2} \mathrm{MeC}_{6} \mathrm{H}_{4}$ & $\mathrm{Rh}\left(\mathrm{PPh}_{3}\right)_{3} \mathrm{Cl}$ & 70 \\
\hline 16 & $\mathrm{CH}_{2} \mathrm{CH}_{2} \mathrm{Ph}$ & $3-\mathrm{C}(\mathrm{O}) \mathrm{MeC}_{6} \mathrm{H}_{4}$ & $\mathrm{Rh}\left(\mathrm{PPh}_{3}\right)_{3} \mathrm{Cl}$ & 64 \\
\hline 17 & $\mathrm{CH}_{2} \mathrm{CH}_{2} \mathrm{Ph}$ & $4-\mathrm{C}(\mathrm{O}) \mathrm{MeC}_{6} \mathrm{H}_{4}$ & $\mathrm{Rh}\left(\mathrm{PPh}_{3}\right)_{3} \mathrm{Cl}$ & 66 \\
\hline 18 & $\mathrm{CH}_{2} \mathrm{CH}_{2} \mathrm{Ph}$ & $\mathrm{Ph}$ & {$\left[\mathrm{Rh}\left(\mathrm{C}_{2} \mathrm{H}_{4}\right)_{2} \mathrm{Cl}\right]_{2}$} & $<10$ \\
\hline 19 & $\mathrm{CH}_{2} \mathrm{CH}_{2} \mathrm{Ph}$ & $4-\mathrm{CF}_{3} \mathrm{C}_{6} \mathrm{H}_{4}$ & {$\left[\mathrm{Rh}\left(\mathrm{C}_{2} \mathrm{H}_{4}\right)_{2} \mathrm{Cl}\right]_{2}$} & $<5$ \\
\hline 20 & $\mathrm{CH}_{2} \mathrm{CH}_{2} \mathrm{Ph}$ & $4-\mathrm{FC}_{6} \mathrm{H}_{4}$ & $\mathrm{Rh}\left(\mathrm{PPh}_{3}\right)_{3} \mathrm{Cl}$ & 58 \\
\hline 21 & $\mathrm{CH}_{2} \mathrm{CH}_{2} \mathrm{Ph}$ & $4-\mathrm{CNC}_{6} \mathrm{H}_{4}$ & {$\left[\mathrm{Rh}\left(\mathrm{C}_{2} \mathrm{H}_{4}\right)_{2} \mathrm{Cl}\right]_{2}$} & $<5$ \\
\hline 22 & $\mathrm{CH}_{2} \mathrm{CH}_{2} \mathrm{Ph}$ & hexyl & {$\left[\mathrm{Rh}\left(\mathrm{C}_{2} \mathrm{H}_{4}\right)_{2} \mathrm{Cl}\right]_{2}$} & $<5$ \\
\hline
\end{tabular}

$a$ Reaction conditions: Phenethyl quinolinyl ketone $15(0.11 \mathrm{mmol})$, arylboronic acid $(0.27 \mathrm{mmol})$, catalyst $(0.01 \mathrm{mmol}$ in $\mathrm{Rh})$, toluene $(2 \mathrm{~mL}), \mathrm{N}_{2}, 140{ }^{\circ} \mathrm{C}, 48 \mathrm{~h} . b$ Value is product formation based on conversion of starting materials by GC/MS analysis. 
Table S8. Reactions with Alkyl Boronic Acids

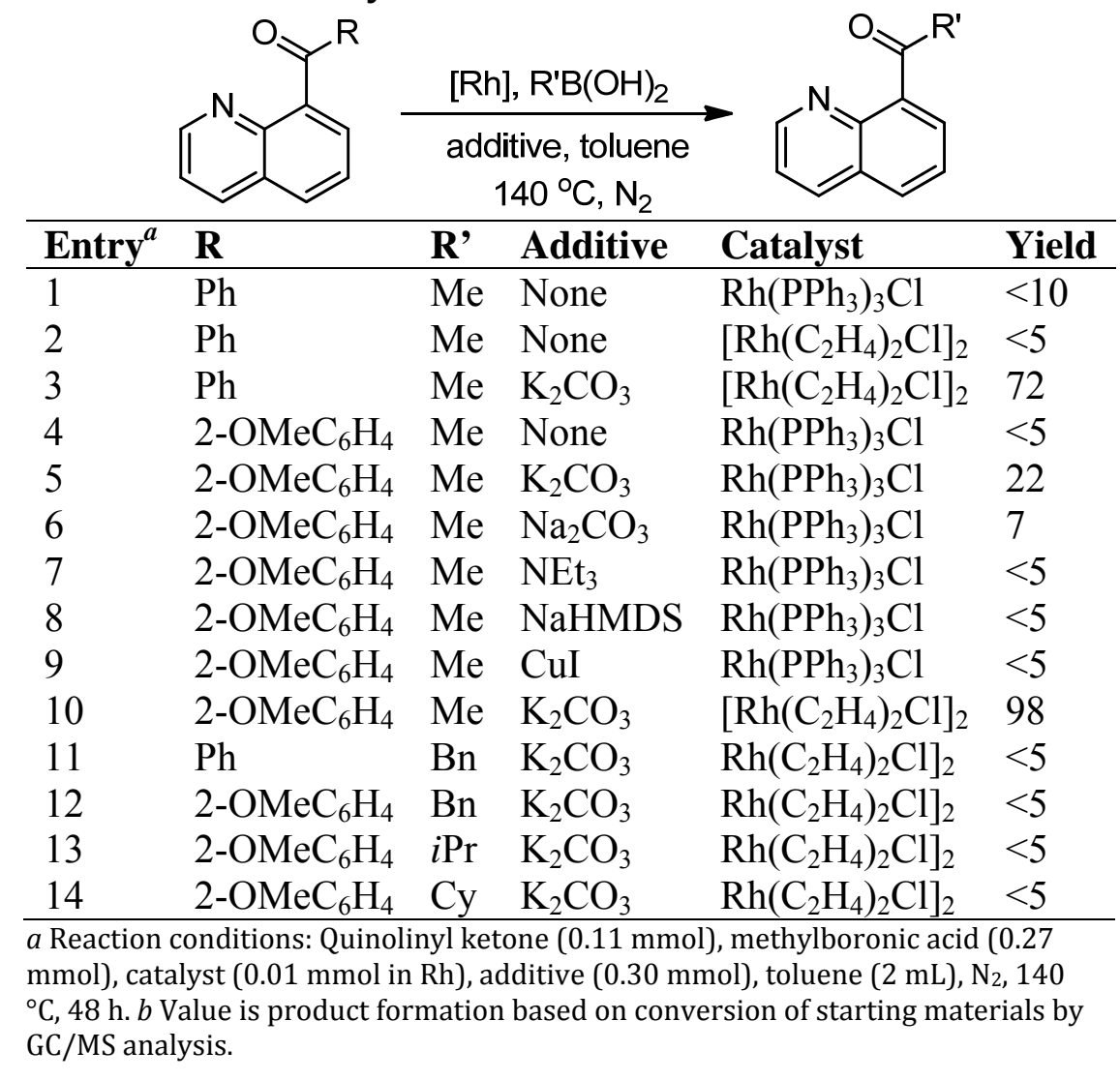

Table S9. Comparison with conditions of Wang and Coworkers

\begin{tabular}{|c|c|c|c|c|c|c|c|}
\hline Entry & $\mathbf{R}$ & $\mathbf{R}^{\prime}$ & Solvent & Atm & Additive & Catalyst & $\begin{array}{l}\text { Yield } \\
\text { (\%) }\end{array}$ \\
\hline $1^{a}$ & $\begin{array}{l}2- \\
\mathrm{OMeC}_{6} \mathrm{H}_{4}\end{array}$ & $4-\mathrm{CF}_{3} \mathrm{C}_{6} \mathrm{H}_{4}$ & $\begin{array}{l}O- \\
\text { xylene }\end{array}$ & air & $\begin{array}{l}\mathrm{K}_{2} \mathrm{CO}_{3}, \mathrm{CuI}, 130 \\
{ }^{\circ} \mathrm{C}\end{array}$ & $\mathrm{Rh}\left(\mathrm{PPh}_{3}\right)_{3} \mathrm{Cl}$ & 9 \\
\hline $2^{b}$ & $\begin{array}{l}2- \\
\mathrm{OMeC}_{6} \mathrm{H}_{4}\end{array}$ & $4-\mathrm{CF}_{3} \mathrm{C}_{6} \mathrm{H}_{4}$ & Toluene & $\mathrm{N}_{2}$ & None, $140^{\circ} \mathrm{C}$ & {$\left[\mathrm{Rh}\left(\mathrm{C}_{2} \mathrm{H}_{4}\right)_{2} \mathrm{Cl}\right]_{2}$} & $98(82)$ \\
\hline $3^{a}$ & $\mathrm{Ph}$ & $\begin{array}{l}\text { 4- } \\
\mathrm{MeOC}_{6} \mathrm{H}_{4}\end{array}$ & $\begin{array}{l}O- \\
\text { xylene }\end{array}$ & Air & $\begin{array}{l}\mathrm{K}_{2} \mathrm{CO}_{3}, \mathrm{CuI}, 130 \\
{ }^{\circ} \mathrm{C}\end{array}$ & $\mathrm{Rh}\left(\mathrm{PPh}_{3}\right)_{3} \mathrm{Cl}$ & 51 \\
\hline $4^{b}$ & $\mathrm{Ph}$ & $\begin{array}{l}4- \\
\mathrm{MeOC}_{6} \mathrm{H}_{4}\end{array}$ & Toluene & $\mathrm{N}_{2}$ & None, $140^{\circ} \mathrm{C}$ & {$\left[\mathrm{Rh}\left(\mathrm{C}_{2} \mathrm{H}_{4}\right)_{2} \mathrm{Cl}\right]_{2}$} & $<5$ \\
\hline
\end{tabular}

$a$ Reaction conditions: Quinolinyl ketone (0.05 mmol), boronic acid (0.125 mmol), CuI (0.1 mmol), $\mathrm{K}_{2} \mathrm{CO}_{3}(0.1$ $\mathrm{mmol}) \mathrm{Rh}\left(\mathrm{PPh}_{3}\right)_{3} \mathrm{Cl}(10 \mathrm{~mol} \%)$ in xylene $(0.5 \mathrm{~mL})$ at $130{ }^{\circ} \mathrm{C}$ in air. $b$ Reaction conditions: Quinolinyl ketone $(0.11$ $\mathrm{mmol})$, methylboronic acid $(0.27 \mathrm{mmol})$, catalyst $(0.01 \mathrm{mmol}$ in $\mathrm{Rh})$, additive $(0.30 \mathrm{mmol})$, in toluene $(2 \mathrm{~mL})$ at $140{ }^{\circ} \mathrm{C}$ under $\mathrm{N}_{2}$. 
<smiles>O=C(c1ccccc1F)c1cccc2cccnc12</smiles>

(2-fluorophenyl)(quinolin-8-yl)methanone (1). Prepared through from 2-fluorobenzaldehyde and 8-bromoquinoline. Product isolated as an orange solid (1.86g, $7.4 \mathrm{mmol}, 55 \%)$ by column chromatography, 9:1 hex:EtOAc, $\mathrm{R}_{\mathrm{f}}=0.19$ (2:1 hex:EtOAc). $\mathrm{mp}=105-106{ }^{\circ} \mathrm{C} .{ }^{1} \mathrm{H}$ 7.96-7.88 (mult, 2H), 7.82 (dd, $J=7.1,1.3 \mathrm{~Hz}, 1 \mathrm{H}), 7.61$ (dd, $J=7.9,7.3 \mathrm{~Hz}, 1 \mathrm{H}), 7.49$ (mult, 1H), 7.38 (dd, $J=8.4,4.2 \mathrm{~Hz}, 1 \mathrm{H}) 7.23$ (t, $J=7.2 \mathrm{~Hz}, 1 \mathrm{H}) .6 .96$ (dd, $J=10.8,8.6$ $\mathrm{Hz}, 1 \mathrm{H}) .{ }^{13} \mathrm{C}\left(100 \mathrm{MHz}, \mathrm{CDCl}_{3}\right) \delta 194.5,163.0,160.4,150.6,145.9,140.5,135.9,134.4$ $(\mathrm{d}, J=10 \mathrm{~Hz}), 130.5,129.9$ (d, $J=274 \mathrm{~Hz}), 128.0,127.7$ (d, $J=10 \mathrm{~Hz}), 126.0,124.1(J$ $=3 \mathrm{~Hz}), 121.5,116.3(\mathrm{~d}, J=23 \mathrm{~Hz})$. IR (diamond ATR) 3073, 3005, 1660, 1606, 1593, 1496, 774. HRMS for $\mathrm{C}_{16} \mathrm{H}_{11} \mathrm{FNO}^{+}$, calcd 252.0819. Found 252.0822.

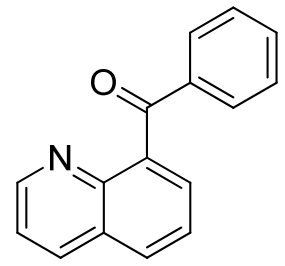

phenyl(quinolin-8-yl)methanone (2). Prepared through the exchange of 2-methoxyquinolinyl ketone 4 with phenylboronic acid using Method A. GC/MS indicates 67\% conversion. Product isolated as a white solid (13 mg, $0.057 \mathrm{mmol}, 52 \%$ ) by column chromatography, 9:1 hex:EtOAc, $\mathrm{R}_{\mathrm{f}}=0.32$ (3:1 hex:EtOAc). Spectral data matches previously reported literature data. ${ }^{2} \mathrm{H} \mathrm{NMR}\left(400 \mathrm{MHz}, \mathrm{CDCl}_{3}\right) \delta 8.83(\mathrm{dd}, J=4.3,1.6 \mathrm{~Hz}, 1 \mathrm{H}), 8.21$ (dd, $J$ $=8.4,1.7 \mathrm{~Hz}, 1 \mathrm{H}), 7.95(\mathrm{~d}, J=8.0 \mathrm{~Hz}, 1 \mathrm{H}), 7.84(\mathrm{~d}, J=7.8 \mathrm{~Hz}, 2 \mathrm{H}), 7.73(\mathrm{~d}, J=6.9 \mathrm{~Hz}$, $1 \mathrm{H}), 7.62(\mathrm{dd}, J=7.7,7.2 \mathrm{~Hz}, 1 \mathrm{H}), 7.54$ (t, $J=7.4 \mathrm{~Hz}, 1 \mathrm{H}), 7.37-7.43$ (mult, $3 \mathrm{H}) .{ }^{13} \mathrm{C}$ $\left(100 \mathrm{MHz}, \mathrm{CDCl}_{3}\right) \delta 197.9,150.8,146.1,139.3,137.7,135.9,133.2,130.1,129.6,128.3$, 128.2, 125.8, 121.6. Probable overlap of peaks at 128.2.

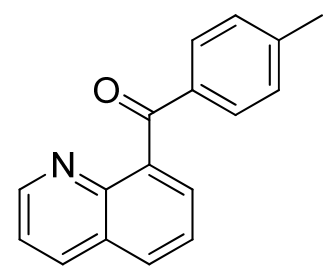

quinolin-8-yl(p-tolyl)methanone (3). Prepared through the exchange of phenethyl ketone 15 with 4-tolyl boronic acid using Method B. GC/MS indicates 62\% conversion. Product isolated as a white solid (13 mg, $0.054 \mathrm{mmol}, 56 \%)$ by column chromatography, 9:1 hex:EtOAc, $\mathrm{R}_{\mathrm{f}}=0.10$ (4:1 hex:EtOAc). $\mathrm{mp}=107-108{ }^{\circ} \mathrm{C} .{ }^{1} \mathrm{H}$ NMR $(400 \mathrm{MHz}$, $\left.\mathrm{CDCl}_{3}\right) \partial 8.75(\mathrm{dd}, J=4.2,1.8 \mathrm{~Hz}, 1 \mathrm{H}), 8.24(\mathrm{dd}, J=8.3,1.8 \mathrm{~Hz}, 1 \mathrm{H}), 7.97(\mathrm{dd}, J=8.2$, 
$1.5 \mathrm{~Hz}, 1 \mathrm{H}), 7.77-7.74$ (mult, 3H), 7.65 (dd, $J=8.1,7.0 \mathrm{~Hz}, 1 \mathrm{H}), 7.44$ (q, $J=7.7 \mathrm{~Hz}$, 1H), 7.24-7.22 (mult, $2 \mathrm{H}), 2.42$ (s, $3 \mathrm{H}) .{ }^{13} \mathrm{C}\left(100 \mathrm{MHz}, \mathrm{CDCl}_{3}\right) \delta$ 197.5, 150.9, 146.2, 144.1, 139.6, 136.0, 135.3, 130.4, 129.5, 129.1, 128.2, 128.1, 125.8, 121.6, 21.8. IR (diamond ATR) 3059, 3028, 3004, 2950, 2921, 1661, 1602, 1593, 1574, 1494, 1468, 1422, 1407. HRMS for $\mathrm{C}_{17} \mathrm{H}_{14} \mathrm{NO}^{+}$, calcd 248.1070. Found 248.1055.

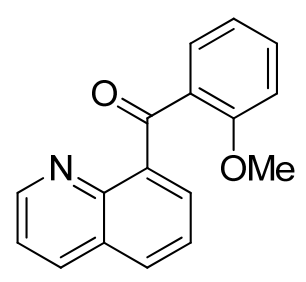

\section{(2-methoxyphenyl)(quinolin-8-yl)methanone (4).}

Prepared via synthesis from 2-methoxybenazldehyde. Product is an orange solid ( $2.51 \mathrm{~g}, 9.4 \mathrm{mmol}, 70 \%$ over two steps) isolated by column chromatography, $\mathrm{R}_{\mathrm{f}}=0.16\left(2: 1\right.$ hex:EtOAc). $\mathrm{mp}=91{ }^{\circ} \mathrm{C} .{ }^{1} \mathrm{H}$

NMR (400 MHz, $\left.\mathrm{CDCl}_{3}\right) \delta 8.80(\mathrm{dd}, J=4.2,1.8 \mathrm{~Hz}, 1 \mathrm{H}), 8.18(\mathrm{dd}, J=8.3,1.8 \mathrm{~Hz}, 1 \mathrm{H})$, $7.90(\mathrm{dd}, J=8.2,1.3 \mathrm{~Hz}, 1 \mathrm{H}), 7.82(\mathrm{dd}, J=7.7,1.8 \mathrm{~Hz}, 1 \mathrm{H}), 7.77$ (dd, $J=7.1,1.4 \mathrm{~Hz}$, 1H), 7.58 (dd, $J=8.0,7.2 \mathrm{~Hz}, 1 \mathrm{H}$ ), 7.48 (mult, 1H), 7.38 (dd, $J=8.3,4.2 \mathrm{~Hz}, 1 \mathrm{H}$ ), 7.03 (t, $J=7.7 \mathrm{~Hz}, 1 \mathrm{H}), 6.87(\mathrm{~d}, J=8.2 \mathrm{~Hz}, 1 \mathrm{H}), 3.36(\mathrm{~s}, 3 \mathrm{H}) .{ }^{13} \mathrm{C}\left(100 \mathrm{MHz}, \mathrm{CDCl}_{3}\right) \delta 196.8$, 159.2, 150.4, 146.0, 141.7, 135.8, 133.8, 131.5, 129.6, 129.2, 128.1, 127.8, 125.9, 121.3, 120.5, 112.1, 55.6. IR (diamond ATR) 1647, 1292, 1244, 1017, 930, 806, 758, 621. HRMS for $\mathrm{C}_{17} \mathrm{H}_{14} \mathrm{NO}_{2}^{+}$, calcd 264.1019. Found 264.1012.<smiles>O=C(c1cc(C(F)(F)F)cc(C(F)(F)F)c1)c1cccc2cccnc12</smiles>
(3,5-bis(trifluoromethyl)phenyl)(quinolin-8-yl)methanone (5). Prepared through the exchange of 2-methoxyquinolinyl ketone 4 with 3,5-bis(trifluoromethyl)phenylboronic acid using Method A. GC/MS indicates $97 \%$ conversion. Product isolated as a white solid (20.0 mg, $0.054 \mathrm{mmol}, 47 \%$ ) by column chromatography,

9:1 hex:EtOAc, $\mathrm{R}_{\mathrm{f}}=0.36$ (3:1 hex:EtOAc). $\mathrm{mp}=124-125{ }^{\circ} \mathrm{C}{ }^{1} \mathrm{H}$ NMR $(400 \mathrm{MHz}$, $\left.\mathrm{CDCl}_{3}\right) \delta 8.74(\mathrm{dd}, J=4.2,1.7 \mathrm{~Hz}, 1 \mathrm{H}), 8.24(\mathrm{dd}, J=8.4,1.7 \mathrm{~Hz}, 1 \mathrm{H}), 8.20(\mathrm{~s}, 2 \mathrm{H}), 8.04$ (dd, $J=8.2,1.3 \mathrm{~Hz}, 1 \mathrm{H}), 8.02$ (s, 1H), 7.84 (dd, $J=7.1,1.3 \mathrm{~Hz}, 1 \mathrm{H}), 7.69$ (dd, $J=8.1$, $7.2 \mathrm{~Hz}, 1 \mathrm{H}), 7.44(\mathrm{dd}, J=8.4,4.2 \mathrm{~Hz}, 1 \mathrm{H}) .{ }^{13} \mathrm{C}\left(100 \mathrm{MHz}, \mathrm{CDCl}_{3}\right) \delta 195.2,150.9,145.9$, 
139.7, 137.2, 136.3, 131.9 (qrt, $J=33 \mathrm{~Hz}), 131.3,129.9$ (qrt, $J=3.3 \mathrm{~Hz}), 129.5,128.3$, 126.3, 126.1 (mult), 123.0 (qrt, $J=273 \mathrm{~Hz}$ ), 122.0. IR (diamond ATR) 1674, 1272, 1181, $1167,1100,764,682 . \mathrm{C}_{18} \mathrm{H}_{10} \mathrm{~F}_{6} \mathrm{NO}^{+}$, calcd 370.0661. Found 370.0680 .

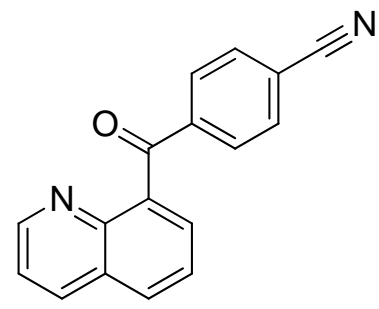

\section{4-(quinoline-8-carbonyl)benzonitrile (6).}

Prepared through the exchange of phenethyl ketone 15 with 4cyanophenylboronic acid using Method B. GC/MS indicates $92 \%$ conversion. Product isolated as a white solid (19.3 mg, 0.075 mmol, 78\%) by column chromatography, 9:1 hex:EtOAc, $\mathrm{R}_{\mathrm{f}}=$

0.12 (4:1 hex:EtOAc). ${ }^{1} \mathrm{H}$ NMR (400 MHz, $\left.\mathrm{CDCl}_{3}\right) \delta 8.79(\mathrm{dd}, J=4.2,1.8 \mathrm{~Hz}, 1 \mathrm{H}), 8.26$ (dd, $J=8.4,1.8 \mathrm{~Hz}, 1 \mathrm{H}$ ), 8.04 (dd, $J=8.2,1.3 \mathrm{~Hz}, 1 \mathrm{H}$ ), 7.89 (mult, 2H), 7.83 (dd, $J=$ 7.1, $1.4 \mathrm{~Hz}, 1 \mathrm{H}), 7.73-7.68$ (mult, $3 \mathrm{H}), 7.45$ (dd, $J=8.3,4.2 \mathrm{~Hz}, 1 \mathrm{H}) .{ }^{13} \mathrm{C}(100 \mathrm{MHz}$, $\left.\mathrm{CDCl}_{3}\right) \delta 196.7,150.9,146.0,141.2,138.0,136.1,132.2,130.7,130.2,129.0,128.2$, 126.1, 121.9, 118.2, 116.1. IR (diamond ATR) 3049, 2963, 2917, 2234, 1666, 1273, 928, 856, 823, 792, 761, 544. HRMS for $\mathrm{C}_{17} \mathrm{H}_{11} \mathrm{~N}_{2} \mathrm{O}^{+}$, calcd 259.0866. Found 259.0872.

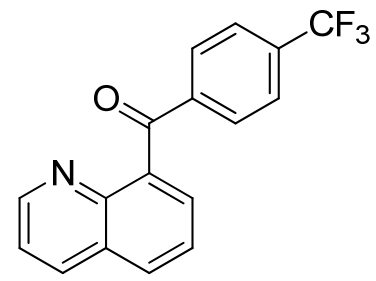

quinolin-8-yl(4-(trifluoromethyl)phenyl)methanone (7).

Prepared through the exchange of 2-methoxyquinolinyl ketone 4 with 4-trifluoromethylphenylboronic acid using Method A. GC/MS indicates $98 \%$ conversion. Product isolated as a white solid (28.1 mg, $0.093 \mathrm{mmol}, 82 \%$ ) by column chromatography, 9:1 hex:EtOAc, $\mathrm{R}_{\mathrm{f}}=0.11$ (2:1 hex:EtOAc). $\mathrm{mp}=99-101{ }^{\circ} \mathrm{C} .{ }^{1} \mathrm{H}$ NMR $\left(400 \mathrm{MHz}, \mathrm{CDCl}_{3}\right) \delta 8.83(\mathrm{dd}, J=4.2,1.8$ $\mathrm{Hz}, 1 \mathrm{H}), 8.26$ (dd, $J=8.35,1.75,1 \mathrm{H}), 8.04$ (dd, $J=8.2,1.4 \mathrm{~Hz}, 1 \mathrm{H}), 7.94(\mathrm{~d}, J=8.1 \mathrm{~Hz}$, 2H), $7.82(\mathrm{dd}, J=7.0,1.4 \mathrm{~Hz}, 1 \mathrm{H}), 7.69$ (dt, $J=8.2,3.5 \mathrm{~Hz}, 3 \mathrm{H}), 7.46$ (dd, $J=8.3,4.2$, $1 \mathrm{H}) .{ }^{13} \mathrm{C}\left(100 \mathrm{MHz}, \mathrm{CDCl}_{3}\right) \delta 197.0,150.9,146.0,140.7,138.5,136.1,134.2$ (q, $J=33.2$ Hz), 130.4, 130.3, 128.7, 128.2, 126.0, 125.4 (q, $J=3.8 \mathrm{~Hz}), 125.0,123.7$ (q, $J=272 \mathrm{~Hz}$ ) 122.3, 121.82. IR (diamond ATR) 3047.3, 2923.4, 2851.1, 1672.0, 1495.0, 1319.6, 1062.8. HRMS for $\mathrm{C}_{17} \mathrm{H}_{11} \mathrm{~F}_{3} \mathrm{NO}^{+}$, calcd 302.0787. Found 302.0803. 


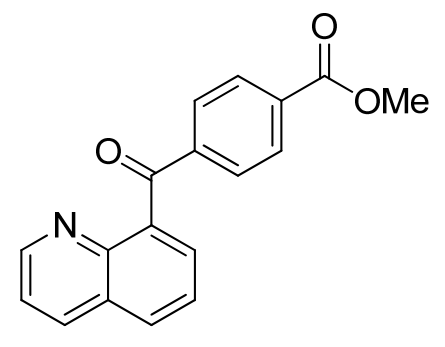

methyl 4-(quinoline-8-carbonyl)benzoate (8).

Prepared through the exchange of 2-methoxyquinolinyl ketone 4 with 4-methoxycarbonylphenylboronic acid using Method A. GC/MS indicates $95 \%$ conversion. Product isolated as a white solid (17.6 mg, $0.060 \mathrm{mmol}, 53 \%$ ) by column chromatography, gradient hex to 4:1 hex:EtOAc, $\mathrm{R}_{\mathrm{f}}=0.08$ (4:1 hex:EtOAc). ${ }^{1} \mathrm{H}$ NMR $\left(400 \mathrm{MHz}, \mathrm{CDCl}_{3}\right) \delta 8.80(\mathrm{dd}, J=4.2,1.7 \mathrm{~Hz}, 1 \mathrm{H}), 8.23(\mathrm{dd}, J=8.3,1.8 \mathrm{~Hz}, 1 \mathrm{H}), 8.08$ 7.99 (mult, 2H), 8.00 (dd, $J=8.1,1.4 \mathrm{~Hz}, 1 \mathrm{H}$ ), 7.88-7.85 (mult, 2H), 7.79 (dd, $J=7.0$, $1.4 \mathrm{~Hz}, 1 \mathrm{H}$ ), 7.66 (dd, $J=8.1,7.1 \mathrm{~Hz}, 1 \mathrm{H}), 7.43$ (dd, $J=8.4,4.2 \mathrm{~Hz}, 1 \mathrm{H}) 3.93$ (s, 3H). ${ }^{13} \mathrm{C}\left(100 \mathrm{MHz}, \mathrm{CDCl}_{3}\right) \delta 197.5,166.4,150.9,146.1,141.2,138.7,136.1,133.7,130.3$, 129.9, 129.5, 128.7, 128.2, 126.0, 121.8, 52.4. HRMS for $\mathrm{C}_{18} \mathrm{H}_{14} \mathrm{NO}_{3}{ }^{+}$, calcd 292.0968. Found 292.0963.

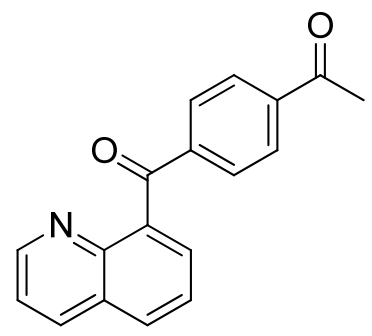

\section{1-(4-(quinoline-8-carbonyl)phenyl)ethanone (9).}

Prepared through the exchange of 2-methoxyquinolinyl ketone 4 with 4-acetylphenylboronic acid using Method A. GC/MS indicates $80 \%$ conversion. Product isolated as a yellowish solid (17.9 mg, $0.065 \mathrm{mmol}, 57 \%$ ) by column chromatography, $4: 1$ hex:EtOAc, $\mathrm{R}_{\mathrm{f}}=0.06$ (4:1 hex:EtOAc). ${ }^{1} \mathrm{H}$ NMR $\left(400 \mathrm{MHz}, \mathrm{CDCl}_{3}\right) \delta 8.80$ (dd, $J=4.2$, $1.8 \mathrm{~Hz}, 1 \mathrm{H}), 8.24$ (dd, $J=8.4,1.8 \mathrm{~Hz}, 1 \mathrm{H}), 8.01$ (dd, $J=8.2,1.3 \mathrm{~Hz}, 1 \mathrm{H}), 7.99-7.96$ (mult, 2H), 7.90-7.88 (mult, 2H), 7.79 (dd, $J=7.1,1.4 \mathrm{~Hz}, 1 \mathrm{H}$ ), 7.66 (dd, $J=8.0,7.1 \mathrm{~Hz}$, 1H), $7.44(\mathrm{dd}, J=8.2,4.2 \mathrm{~Hz}, 1 \mathrm{H}), 2.63(\mathrm{~s}, 3 \mathrm{H}) .{ }^{13} \mathrm{C}\left(100 \mathrm{MHz}, \mathrm{CDCl}_{3}\right) \delta$ 197.6, 197.4, 150.9, 146.1, 141.2, 140.0, 138.7, 136.1, 130.3, 130.2, 128.7, 128.2, 128.2, 128.0, 121.8, 26.9. HRMS for $\mathrm{C}_{18} \mathrm{H}_{14} \mathrm{NO}_{2}{ }^{+}$, calcd 276.1019. Found 276.1013.<smiles>O=C(c1cccc([N+](=O)[O-])c1)c1cccc2cccnc12</smiles>

(3-nitrophenyl)(quinolin-8-yl)methanone (10). 
Prepared through the exchange of 2-methoxyquinolinyl ketone 4 with 3nitrophenylboronic acid using Method A. GC/MS indicates 74\% conversion. Product isolated as a white solid (10 mg, $0.036 \mathrm{mmol}, 30 \%$ ) by column chromatography, 9:1 hex:EtOAc, $\mathrm{R}_{\mathrm{f}}=0.11\left(2: 1\right.$ hex:EtOAc). ${ }^{1} \mathrm{H}$ NMR $\left(400 \mathrm{MHz}, \mathrm{CDCl}_{3}\right) \delta 8.76$ (dd, $J=4.0$, $1.5 \mathrm{~Hz}, 1 \mathrm{H}), 8.56$ (s, 1H), 8.39 (d, $J=7.4 \mathrm{~Hz}, 1 \mathrm{H}), 8.25$ (dd, $J=8.4,1.4 \mathrm{~Hz}, 1 \mathrm{H}), 8.16$ (d, $J=7.7 \mathrm{~Hz}, 1 \mathrm{H}), 8.04$ (d, $J=7.1 \mathrm{~Hz}, 1 \mathrm{H}), 7.83(\mathrm{~d}, J=7.5 \mathrm{~Hz}, 1 \mathrm{H}), 7.69$ (t, $J=8.1 \mathrm{~Hz}$, $1 \mathrm{H}), 7.61(\mathrm{t}, J=7.9 \mathrm{~Hz}, 1 \mathrm{H}), 7.44(\mathrm{q}, J=4.2 \mathrm{~Hz}, 1 \mathrm{H}) .{ }^{13} \mathrm{C}\left(100 \mathrm{MHz}, \mathrm{CDCl}_{3}\right) \delta 195.9$, $150.9,148.3,145.9,139.5,137.8,136.2,135.3,130.9,129.5,129.1,128.3,127.2,126.2$, 124.8, 121.9. HRMS for $\mathrm{C}_{16} \mathrm{H}_{11} \mathrm{~N}_{2} \mathrm{O}_{2}{ }^{+}$, calcd 279.0764. Found 279.0778.

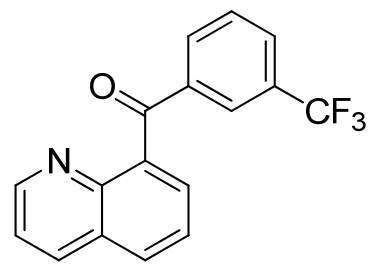

\section{(3-(trifluoromethyl)phenyl) (quinolin-8-yl)methanone} (11).

Prepared through the exchange of 2-methoxyquinolinyl ketone 4 with 3-trifluoromethylphenylboronic acid using Method A.

GC/MS indicates 77\% conversion. Product isolated as a white solid (15.8 mg, 0.052 mmol, $46 \%)$ by column chromatography, $\mathrm{R}_{\mathrm{f}}=0.21\left(2: 1\right.$ hex:EtOAc). $\mathrm{mp}=77-79{ }^{\circ} \mathrm{C} .{ }^{1} \mathrm{H}$ NMR (400 MHz, $\left.\mathrm{CDCl}_{3}\right) \delta 8.81(\mathrm{dd}, J=4.2,1.8 \mathrm{~Hz}, 1 \mathrm{H}), 8.24(\mathrm{dd}, J=8.4,1.8 \mathrm{~Hz}, 1 \mathrm{H})$, 8.14 (s, 1H), 8.02 (dd, $J=8.2,1.4 \mathrm{~Hz}, 1 \mathrm{H}), 7.94$ (d, $J=7.8 \mathrm{~Hz}, 1 \mathrm{H}), 7.80$ (m, 2H), 7.67 (m, $1 \mathrm{H}), 7.48(\mathrm{~m}, 2 \mathrm{H}) .{ }^{13} \mathrm{C}\left(100 \mathrm{MHz}, \mathrm{CDCl}_{3}\right) \delta 196.6,150.9,148.7,146.0,138.5,138.4$, 137.2, 136.1, 133.4, 130.4, 129.5, 128.9, 128.7, 128.3, 126.6, 126.1, 121.8. IR (diamond ATR) $1666,1331,1270,1113,1068,795,697,626$. HRMS for $\mathrm{C}_{17} \mathrm{H}_{11} \mathrm{~F}_{3} \mathrm{NO}^{+}$, calcd 302.0787. Found 302.0782.

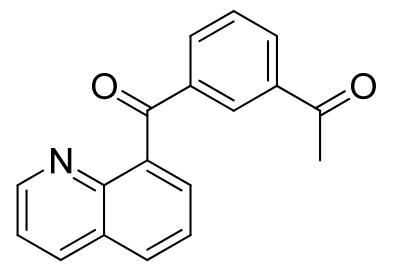

\section{1-(3-(quinoline-8-carbonyl)phenyl)ethanone (12).}

Prepared through the exchange of 2-methoxyquinolinyl ketone 4 with 3-acetylphenylboronic acid boronic acid using Method A. GC/MS indicates $85 \%$ conversion. Product isolated as a yellowish solid (12.5 mg, $0.046 \mathrm{mmol}, 40 \%$ ) by column chromatography, gradient from hexane to 4:1 hex:EtOAc, $\mathrm{R}_{\mathrm{f}}=0.10$ (4:1 hex:EtOAc). ${ }^{1} \mathrm{H}$ NMR (400 MHz, $\left.\mathrm{CDCl}_{3}\right) \delta 8.80$ 
(dd, $J=4.2,1.8,1 \mathrm{H}), 8.46$ (t, $J=1.5 \mathrm{~Hz}, 1 \mathrm{H}), 8.24$ (dd, $J=8.4,1.7 \mathrm{~Hz}, 1 \mathrm{H}), 8.15$ (dt, $J=$ 7.8, $1.3 \mathrm{~Hz}, 1 \mathrm{H}), 8.01$ (dd, $J=8.2,1.4 \mathrm{~Hz}, 1 \mathrm{H}), 7.94$ (dt, $J=7.8,1.3 \mathrm{~Hz}, 1 \mathrm{H}), 7.79$ (dd, $J$ $=7.0,1.4 \mathrm{~Hz}, 1 \mathrm{H}), 7.66(\mathrm{dd}, J=8.1,7.1 \mathrm{~Hz}, 1 \mathrm{H}), 7.50(\mathrm{t}, J=7.72 \mathrm{~Hz}, 1 \mathrm{H}), 7.44(\mathrm{dd}, J=$ 8.2, 4.2 Hz, 1H), $2.60(\mathrm{~s}, 3 \mathrm{H}) .{ }^{13} \mathrm{C}\left(100 \mathrm{MHz}, \mathrm{CDCl}_{3}\right) \delta 197.4,197.2,150.9,146.1,138.7$, 138.3, 137.3, 136.1, 134.7, 132.4, 130.3, 129.6, 128.7, 128.6, 128.3, 126.0, 121.8, 26.7. HRMS for $\mathrm{C}_{18} \mathrm{H}_{13} \mathrm{NNaO}_{2}{ }^{+}$, calcd 298.0838. Found 298.0828.

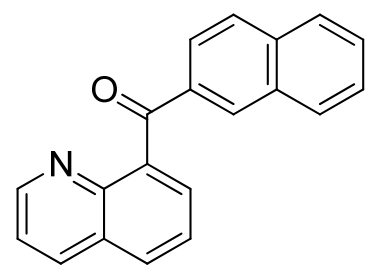

\section{naphthalen-2-yl(quinolin-8-yl)methanone (13).}

Prepared through the exchange of 2-methoxyquinolinyl ketone 4 with 2-naphthylboronic acid using Method A. GC/MS indicates $88 \%$ conversion. Product isolated as a white solid (10.6 mg, 0.038 mmol, 33\%) by column chromatography, 9:1 hex:EtOAc, $\mathrm{R}_{\mathrm{f}}=0.30$ (2:1 hex:EtOAc). ${ }^{1} \mathrm{H}$ NMR (400 MHz, $\left.\mathrm{CDCl}_{3}\right) \delta 8.82(\mathrm{dd}, J=4.2,1.71 \mathrm{H}), 8.24(\mathrm{dd}, J=8.31,1.62 \mathrm{~Hz}, 1 \mathrm{H})$, $8.192(\mathrm{~s}, 1 \mathrm{H}), 8.07$ (dd, $J=8.6,1.6 \mathrm{~Hz}, 1 \mathrm{H}), 8.00(\mathrm{dd}, J=8.2,1.1 \mathrm{~Hz}, 1 \mathrm{H}), 7.87$ (t, $J=$ $8.7,2 \mathrm{H}), 7.80$ (d, $J=7.0,1.2,1 \mathrm{H}), 7.77$ (d, $J=8.3,1 \mathrm{H}), 7.67$ (t, $J=7.97,1 \mathrm{H}), 7.57$ (t, $J$ $=7.6 \mathrm{~Hz}, 1 \mathrm{H}), 7.46(\mathrm{t}, J=7.2 \mathrm{~Hz}, 1 \mathrm{H}), 7.41(\mathrm{q}, J=4.2 \mathrm{~Hz}, 1 \mathrm{H}) .{ }^{13} \mathrm{C}\left(100 \mathrm{MHz}, \mathrm{CDCl}_{3}\right) \delta$ $197.9,150.9,146.3,139.5,136.0,135.8,135.3,132.8,132.5,129.8,129.7,128.5,128.4$, 128.3, 128.2, 127.8, 126.5, 125.9, 125.2, 121.7. IR (diamond ATR) 3057, 2965, 2929 , 1649, 1594. HRMS for $\mathrm{C}_{20} \mathrm{H}_{14} \mathrm{NO}^{+}$, calcd 284.1070. Found 284.1075.

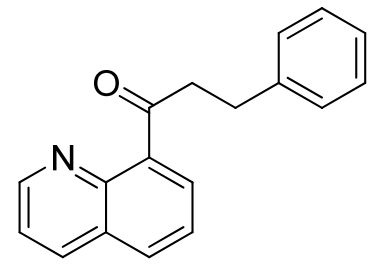

\section{3-phenyl-1-(quinolin-8-yl)propan-1-one (15).}

Prepared from hydrocinnamaldehyde. Product isolated as a yellow solid (1.39g, $5.3 \mathrm{mmol}, 41 \%)$ by column chromatography, 9:1 hex:EtOAc, $\mathrm{R}_{\mathrm{f}}=0.39$ (2:1 hex:EtOAc). ${ }^{1} \mathrm{H}$ NMR $(400 \mathrm{MHz}$, $\left.\mathrm{CDCl}_{3}\right) \delta 8.99(\mathrm{dd}, J=3.9,1.4 \mathrm{~Hz}, 1 \mathrm{H}), 8.21(\mathrm{dd}, J=8.31,1.1 \mathrm{~Hz}, 1 \mathrm{H}), 7.94(\mathrm{~d}, J=8.1$ $\mathrm{Hz}, 1 \mathrm{H}), 7.90$ (d, $J=7.1 \mathrm{~Hz}, 1 \mathrm{H}), 7.60$ (t, $J=7.7 \mathrm{~Hz}, 1 \mathrm{H}), 7.46$ (q, $J=4.2 \mathrm{~Hz}, 1 \mathrm{H}), 7.32-$ 7.31 (mult, 3H), 7.25-7.23 (mult, 2H), 3.75 (t, $J=7.6 \mathrm{~Hz}, 2 \mathrm{H}$ ), 3.19 (t, $J=7.7 \mathrm{~Hz}, 2 \mathrm{H}$ ). ${ }^{13} \mathrm{C}\left(100 \mathrm{MHz}, \mathrm{CDCl}_{3}\right) \delta 205.6,150.5,145.5,141.6,139.7,136.2,131.0,129.2,128.5$, 
128.4, 128.3, 126.0, 125.9, 121.4, 46.4, 30.5. IR (diamond ATR) 3027, 2918, 2863, 1678, 794, 754, 733, 699. HRMS for $\mathrm{C}_{18} \mathrm{H}_{16} \mathrm{NO}^{+}$, calcd 262.1226. Found 262.1220.

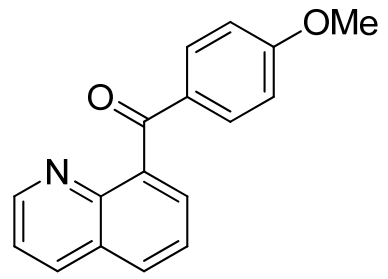

\section{(4-methoxyphenyl)(quinolin-8-yl)methanone (16).}

Prepared through the exchange of phenethyl quinolinyl ketone 15 with 4-methoxyphenyl boronic acid using Method B. GC/MS indicates $51 \%$ conversion. Product isolated as an orange solid (13.1 mg, $0.050 \mathrm{mmol}, 33 \%$ ) by column chromatography, $\mathrm{R}_{\mathrm{f}}=0.19$ (3:1 hex:EtOAc). ${ }^{1} \mathrm{H}$ NMR (400 MHz, $\left.\mathrm{CDCl}_{3}\right) \delta 8.87(\mathrm{~d}, J=3.9,1 \mathrm{H}), 8.20(\mathrm{~d}, J=8.2 \mathrm{~Hz}, 1 \mathrm{H}), 7.94(\mathrm{~d}, J=8.3$ $\mathrm{Hz}, 1 \mathrm{H}), 7.82(\mathrm{~d}, J=8.4 \mathrm{~Hz}, 2 \mathrm{H}), 7.71(\mathrm{~d}, J=7.0 \mathrm{~Hz}, 1 \mathrm{H}), 7.61,(\mathrm{t}, J=7.5 \mathrm{~Hz}, 1 \mathrm{H}), 7.41$ $(\mathrm{dd}, J=8.1,4.1 \mathrm{~Hz}, 1 \mathrm{H}), 6.88(\mathrm{~d}, J=8.0,2 \mathrm{H}), 3.84(\mathrm{~s}, 3 \mathrm{H}) .{ }^{13} \mathrm{C}\left(100 \mathrm{MHz}, \mathrm{CDCl}_{3}\right) \delta$ $196.3,163.6,150.7,145.8,139.5,136.0,132.5,130.8,129.3,128.1,127.9,125.7,121.5$, 113.5, 55.3. IR (diamond ATR) 3021, 1681, 1584, 1468, 1432, 1280, 773. HRMS for $\mathrm{C}_{17} \mathrm{H}_{14} \mathrm{NO}_{2}{ }^{+}$, calcd 264.1019 Found 264.1027.

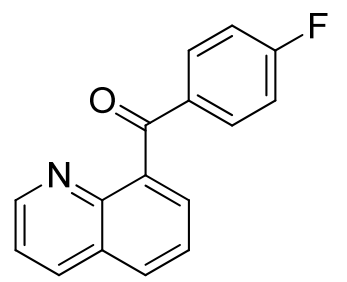

\section{(4-fluorophenyl)(quinolin-8-yl)methanone (18).}

Prepared through the exchange of phenethyl quinolinyl ketone 15 with 4-fluorophenylboronic acid using Method B. GC/MS indicates $58 \%$ conversion. Product isolated as a peach solid $(8.6 \mathrm{mg}, 0.034$ mmol, $36 \%)$ by column chromatography, $\mathrm{R}_{\mathrm{f}}=0.18\left(3: 1\right.$ hex:EtOAc) $. \mathrm{mp}=105^{\circ} \mathrm{C} .{ }^{1} \mathrm{H}$ $\operatorname{NMR}\left(400 \mathrm{MHz}, \mathrm{CDCl}_{3}\right) \delta 8.84(\mathrm{dd}, J=4.2,1.8 \mathrm{~Hz}, 1 \mathrm{H}), 8.23(\mathrm{dd}, J=8.4,1.8 \mathrm{~Hz}, 1 \mathrm{H})$, 7.97 (dd, $J=8.2,1.4$ Hz, 1H), 7.88-7.83 (mult, 2H), 7.74 (dd, J= 7.0, 1.4 Hz, 1H), 7.64 $(\mathrm{dd}, J=8.2,7.2 \mathrm{~Hz}, 1 \mathrm{H}), 7.43(\mathrm{dd}, J=8.2,4.1 \mathrm{~Hz}, 1 \mathrm{H}), 7.08(\mathrm{t}, J=8.7 \mathrm{~Hz}, 2 \mathrm{H}) .{ }^{13} \mathrm{C}(100$ $\left.\mathrm{MHz}, \mathrm{CDCl}_{3}\right) \delta 196.5,166.0(\mathrm{~d}, J=256 \mathrm{~Hz}), 151.1,148.8,146.2,139.2,136.2,133.0(\mathrm{~d}$, $J=10 \mathrm{~Hz}), 130.0,128.4,126.1,121.9,121.3,115.6(\mathrm{~d}, J=24 \mathrm{~Hz})$. IR (diamond ATR) 1682, 1573, 1280, 1224, 793, 749, 601, 474. HRMS for $\mathrm{C}_{16} \mathrm{H}_{11} \mathrm{FNO}^{+}$, calcd 252.0819. Found 252.0831. 


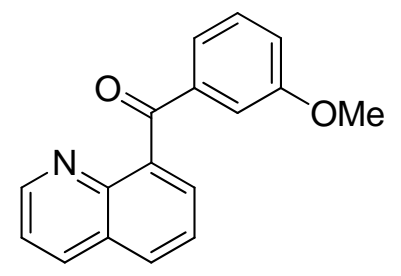

\section{(3-methoxyphenyl)(quinolin-8-yl)methanone (19).}

Prepared through the exchange of phenethyl quinolinyl ketone 15 with 3-methoxyphenyl boronic acid using Method B. GC/MS indicates $73 \%$ conversion. Product isolated as an orange solid (13.1 mg, $0.050 \mathrm{mmol}, 52 \%)$ by column chromatography, $\mathrm{R}_{\mathrm{f}}=0.18$ (3:1 hex:EtOAc). ${ }^{1} \mathrm{H}$ NMR (400 MHz, $\left.\mathrm{CDCl}_{3}\right) \delta 8.80(\mathrm{dd}, J=4.1,1.5 \mathrm{~Hz}, 1 \mathrm{H}), 8.15(\mathrm{dd}, J=8.3,1.6 \mathrm{~Hz}, 1 \mathrm{H})$, $7.90(\mathrm{~d}, J=8.2 \mathrm{~Hz}, 1 \mathrm{H}), 7.70$ (d, $J=7.0 \mathrm{~Hz}, 1 \mathrm{H}), 7.57$ (dd, $J=8.2,7.0 \mathrm{~Hz}, 1 \mathrm{H}), 7.54$, (s, 1H), 7.35 (dd, $J=8.1,4.1 \mathrm{~Hz}, 1 \mathrm{H}), 7.28-7.21$ (mult, 2H), 7.07 (dt, $J=7.3,2.6 \mathrm{~Hz}, 1 \mathrm{H}$ ), $3.77(\mathrm{~s}, 3 \mathrm{H}) .{ }^{13} \mathrm{C}\left(100 \mathrm{MHz}, \mathrm{CDCl}_{3}\right) \delta 197.5,159.4,150.6,145.8,139.0,138.9,135.8$, 129.5, 129.1, 127.9, 127.8, 125.6, 123.4, 121.4, 119.6, 113.5, 55.1. IR (diamond ATR) $3011,1689,1573,1475,1280,1224,793,749,601$. HRMS for $\mathrm{C}_{17} \mathrm{H}_{14} \mathrm{NO}_{2}{ }^{+}$, calcd 264.1019 Found 264.1032. 
${ }^{1} \mathrm{H}$ NMR spectrum for $\mathbf{1}$

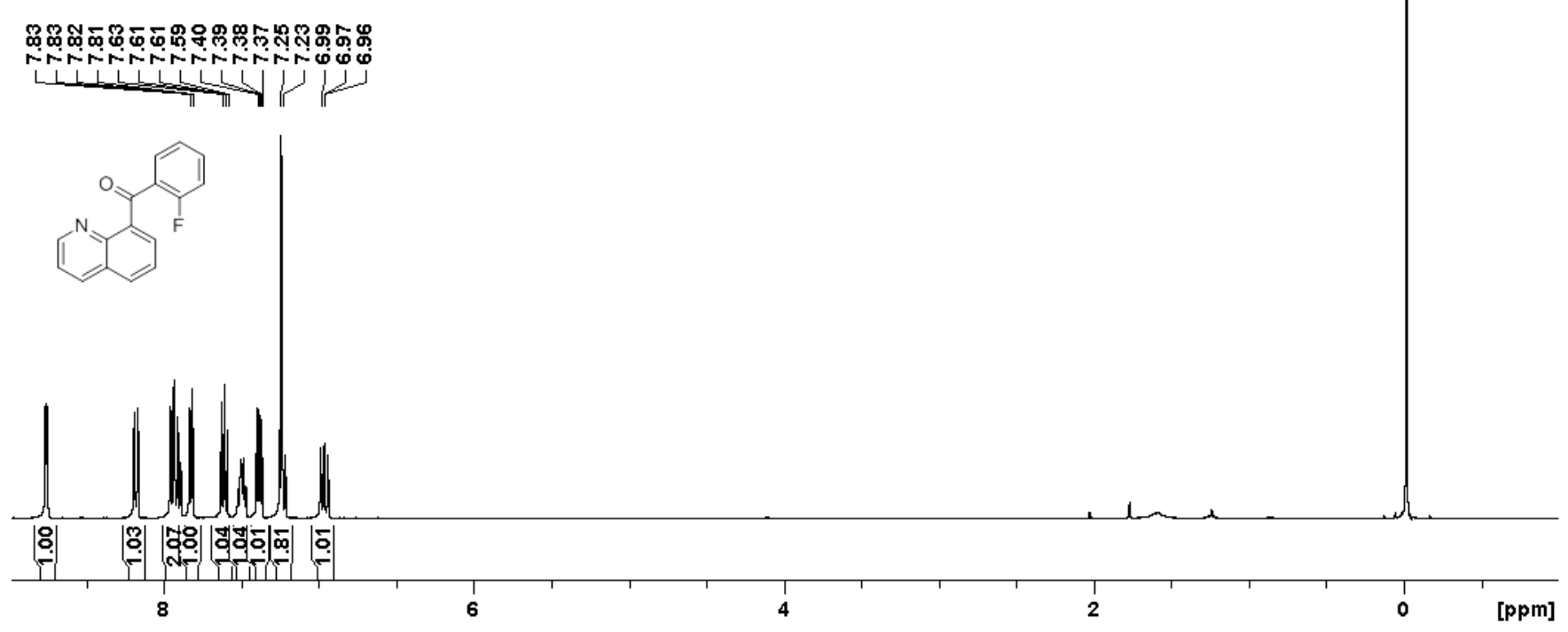

${ }^{13} \mathrm{C}$ NMR spectrum for $\mathbf{1}$

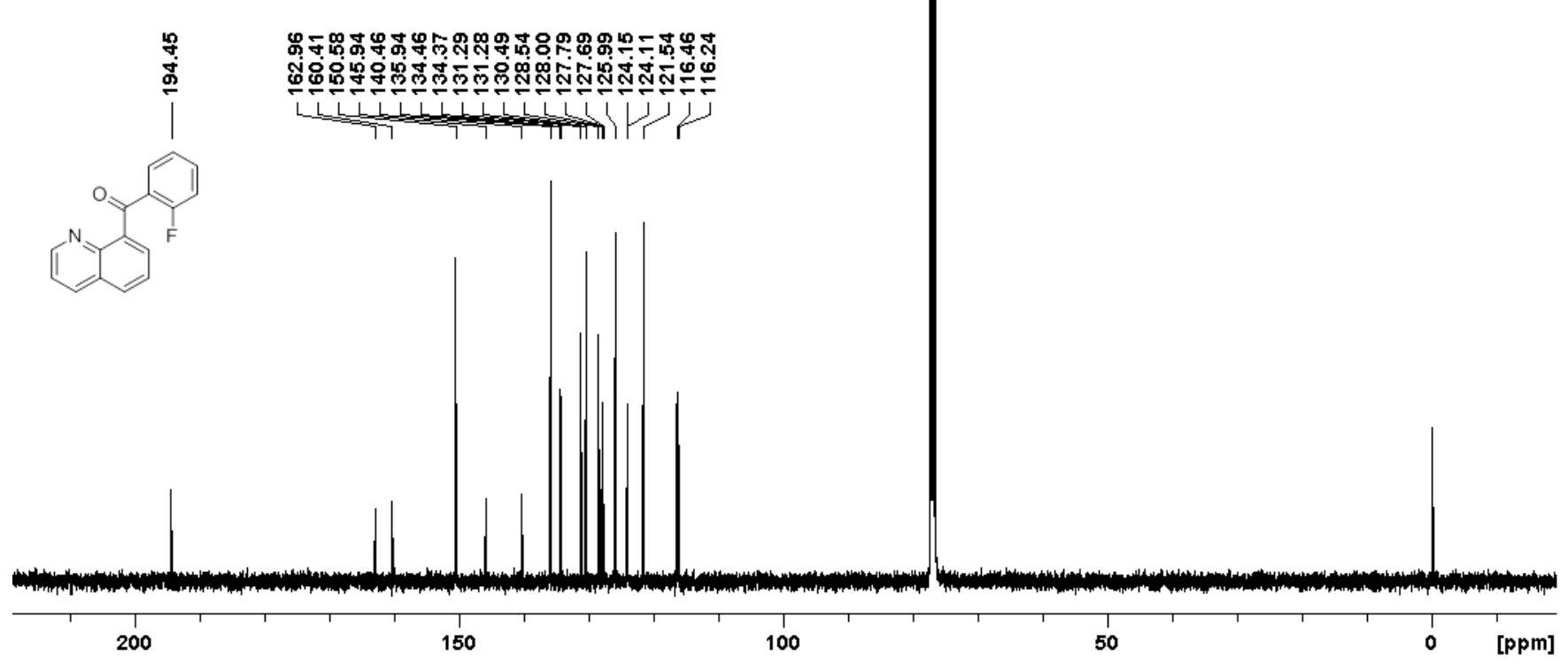


${ }^{1} \mathrm{H}$ NMR spectrum for $\mathbf{2}$

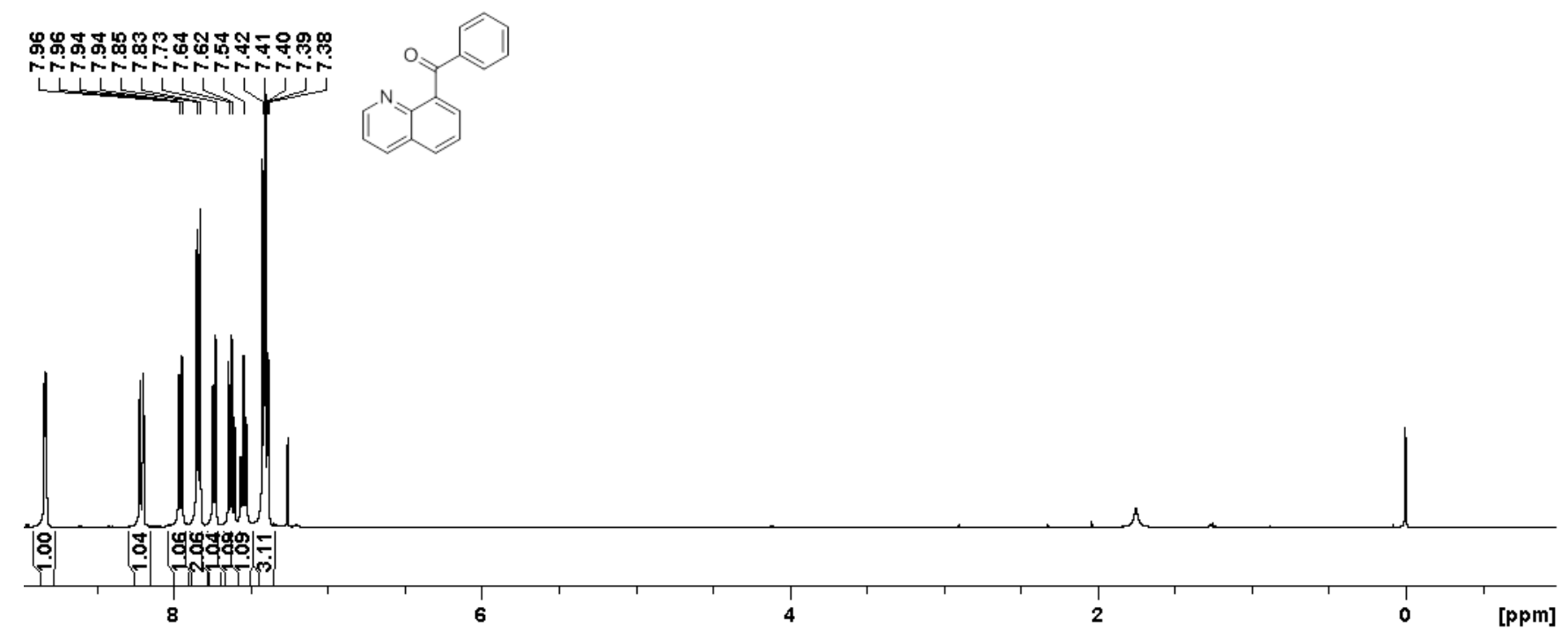

${ }^{13} \mathrm{C}$ NMR spectrum for 2

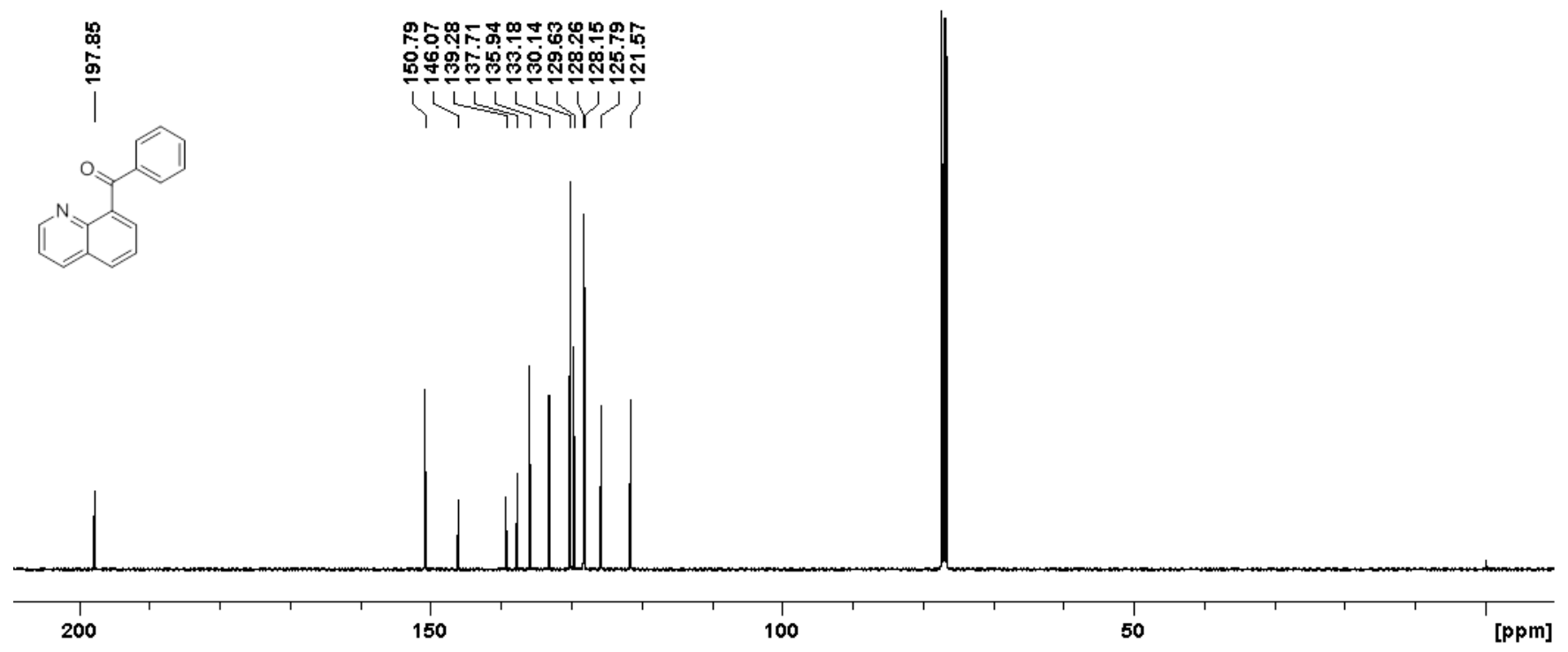


${ }^{1} \mathrm{H}$ NMR spectrum for $\mathbf{3}$

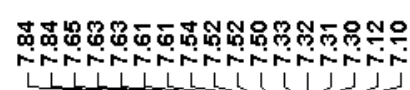

$L \perp \perp \perp \perp \perp<+L$
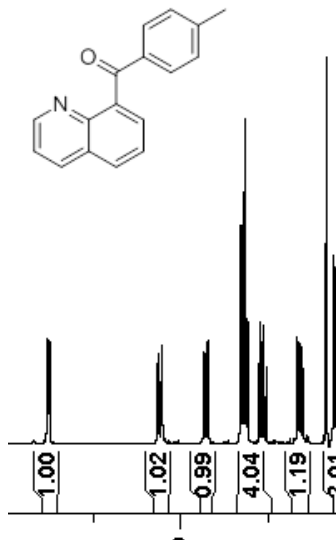

8

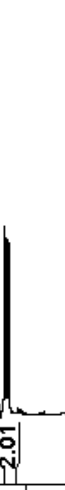

卫

${ }^{13} \mathrm{C}$ NMR spectrum for 3

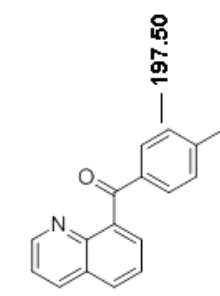

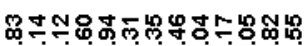

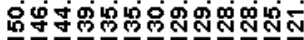

47
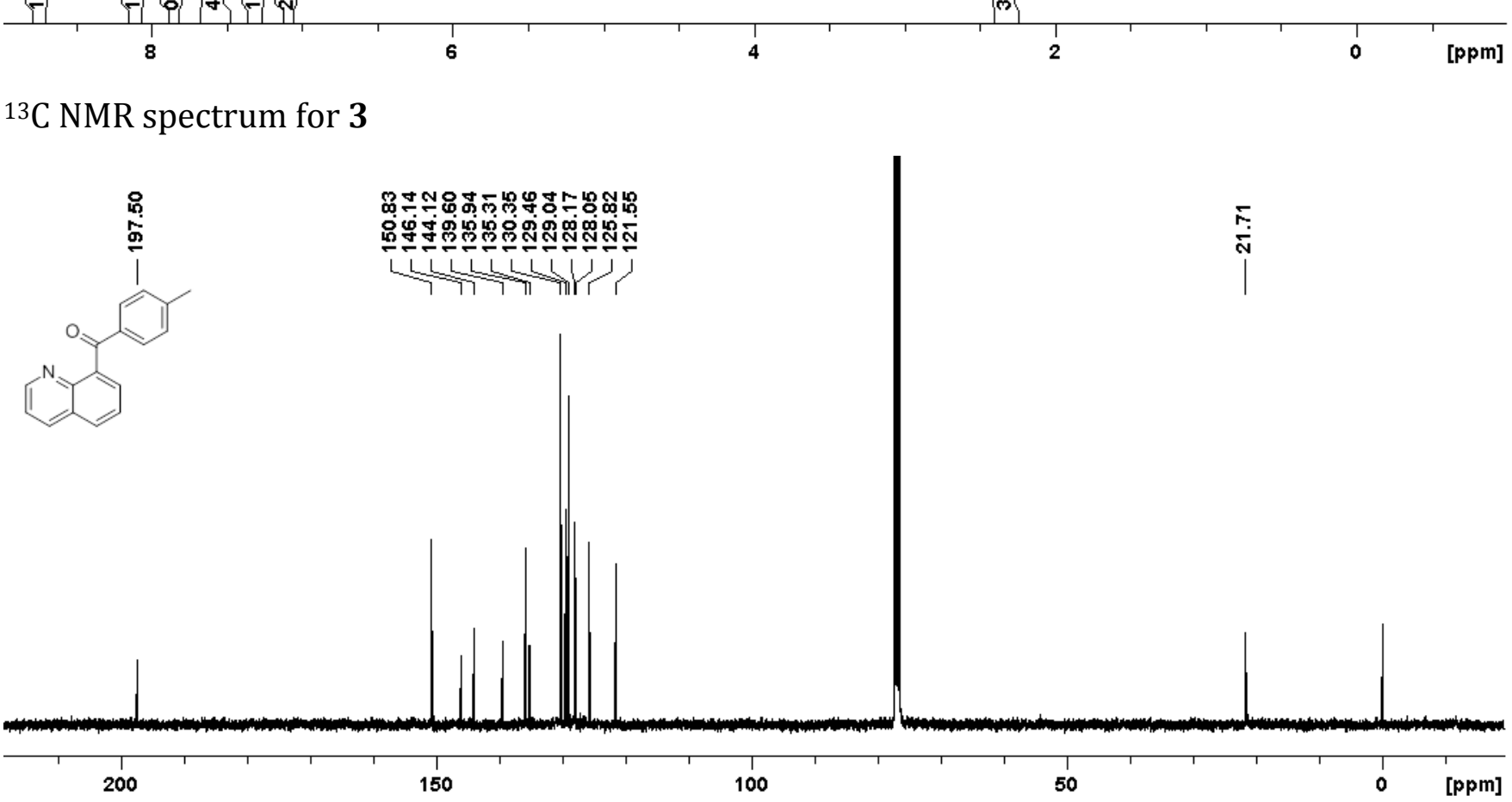
${ }^{1} \mathrm{H}$ NMR spectrum for 4

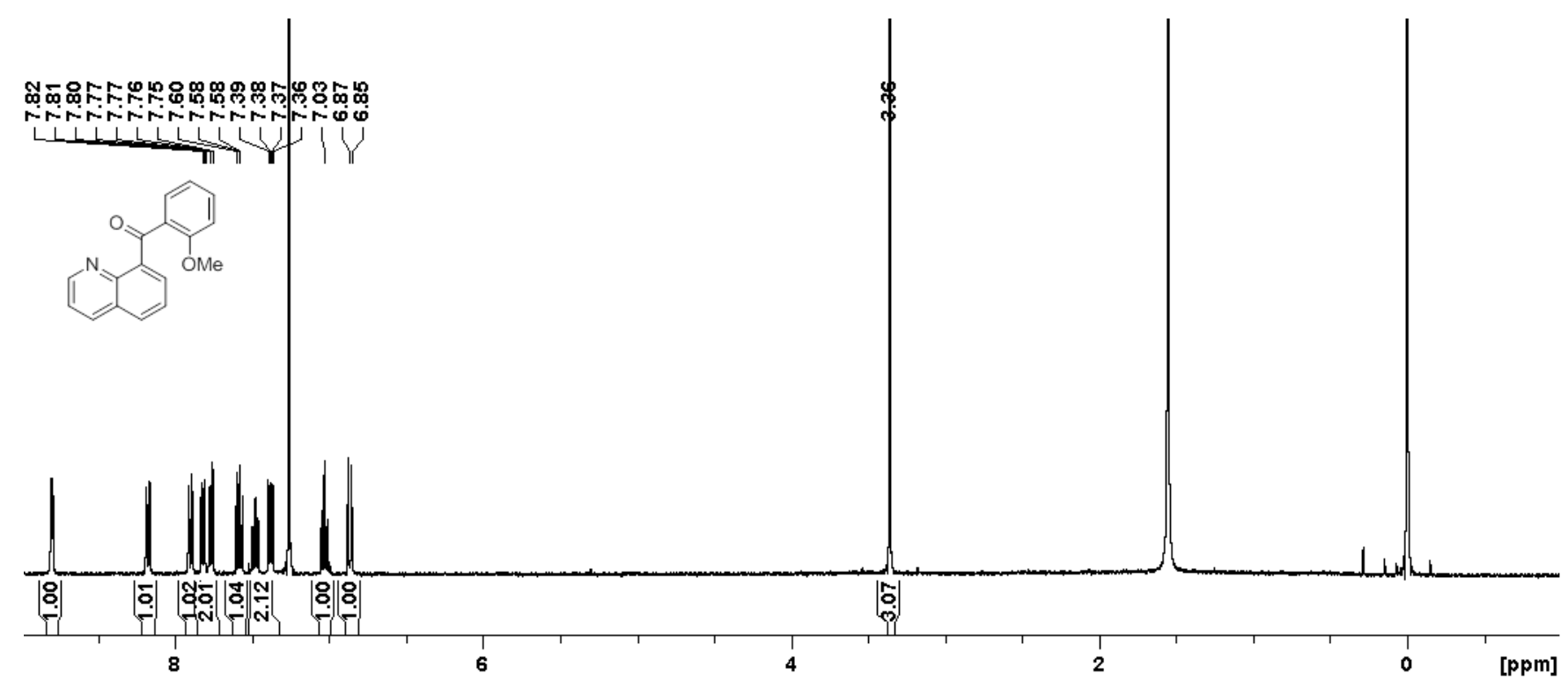

${ }^{13} \mathrm{C}$ NMR spectrum for 4

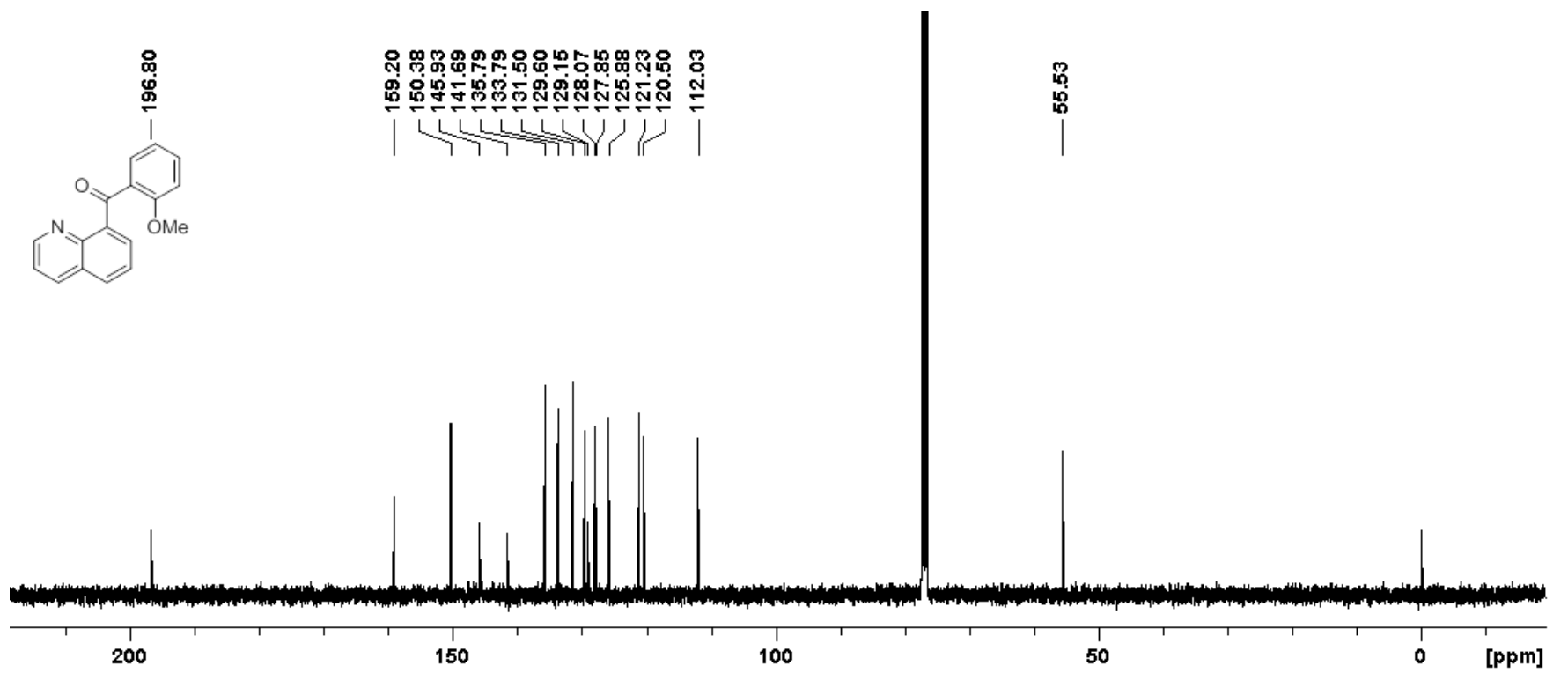


${ }^{1} \mathrm{H}$ NMR spectrum for $\mathbf{5}$

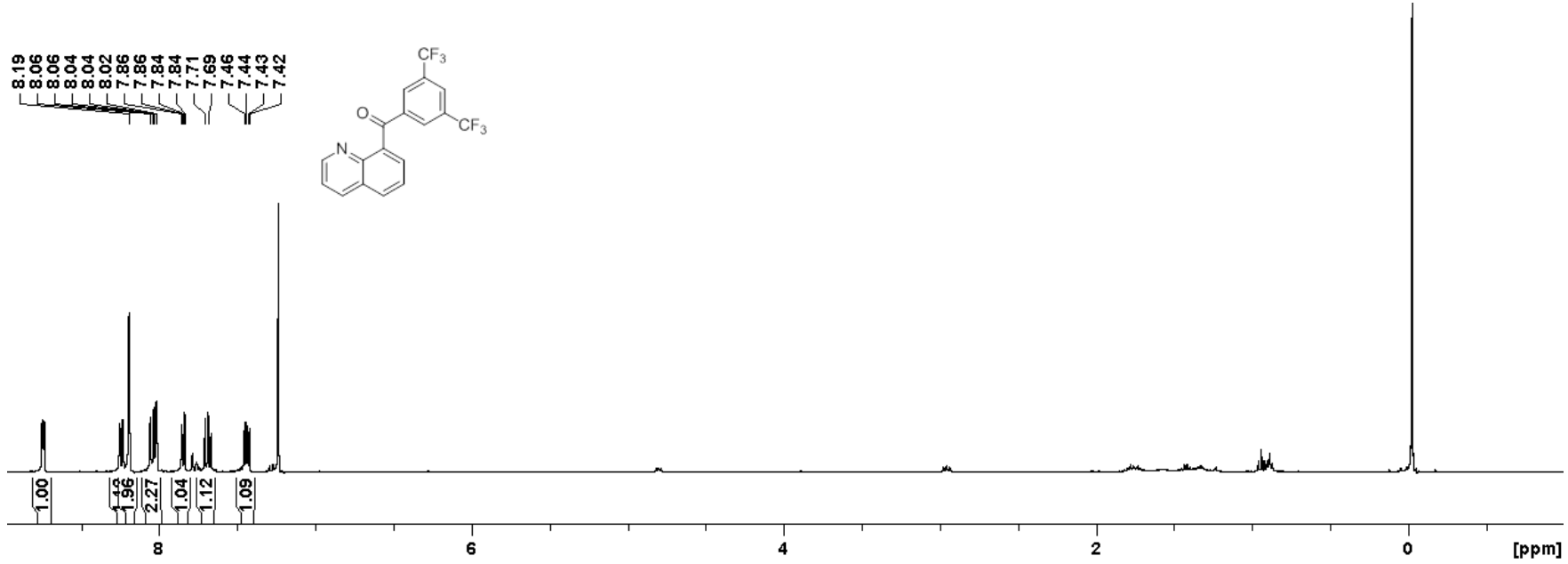

${ }^{13} \mathrm{C}$ NMR spectrum for $\mathbf{5}$

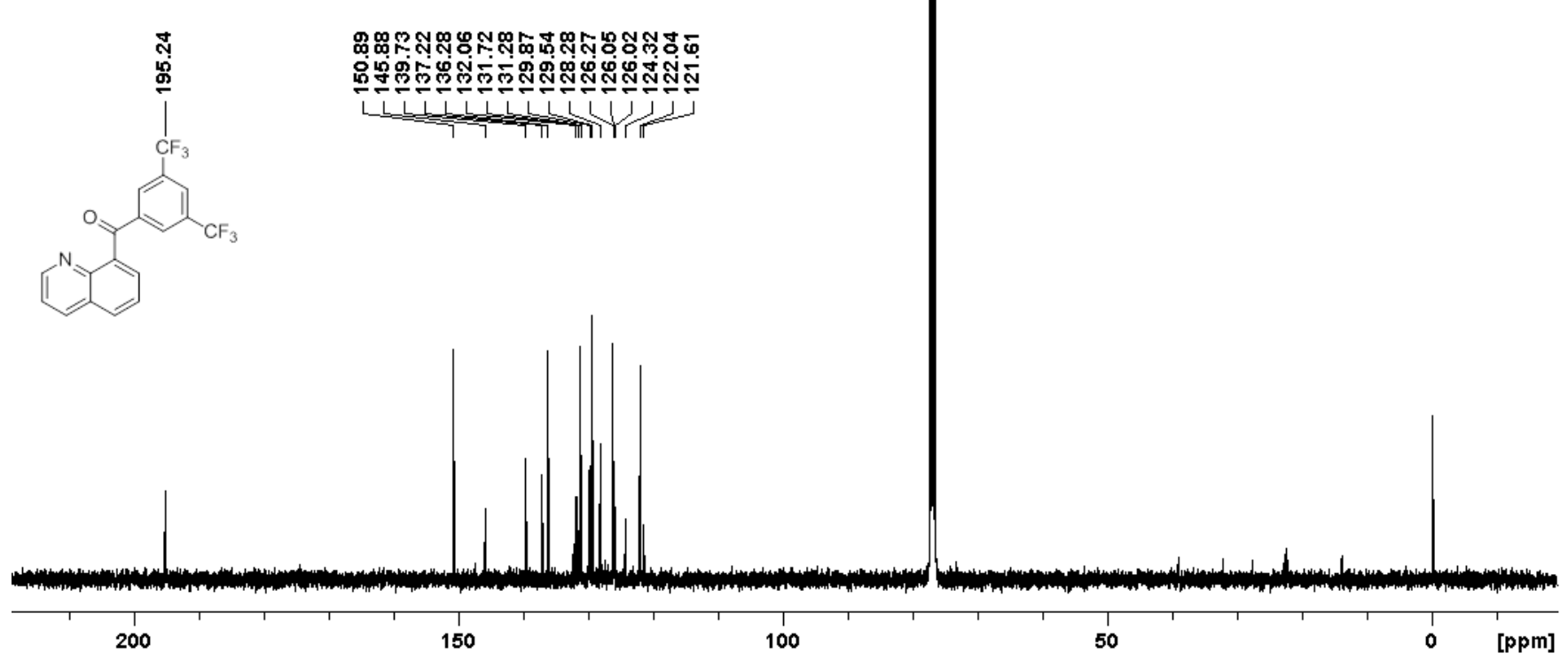


${ }^{1} \mathrm{H}$ NMR spectrum for 6
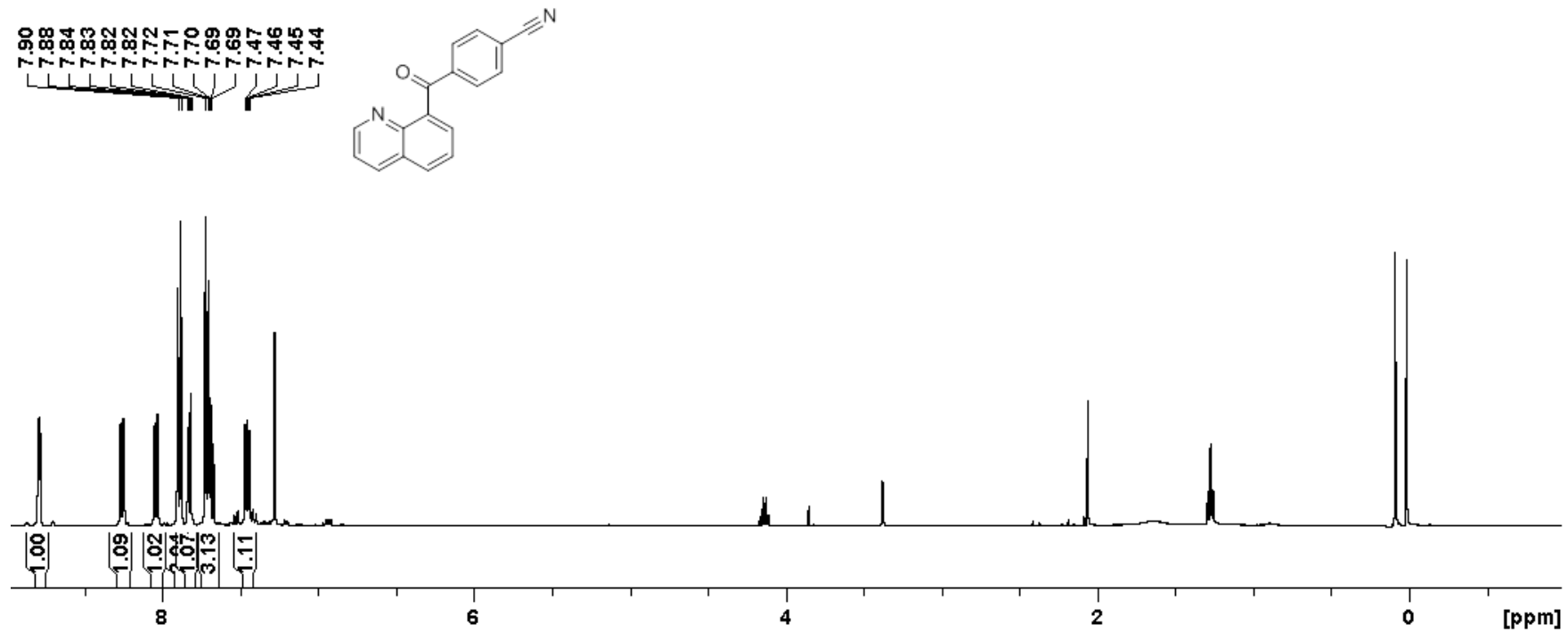

${ }^{13} \mathrm{C}$ NMR spectrum for 6

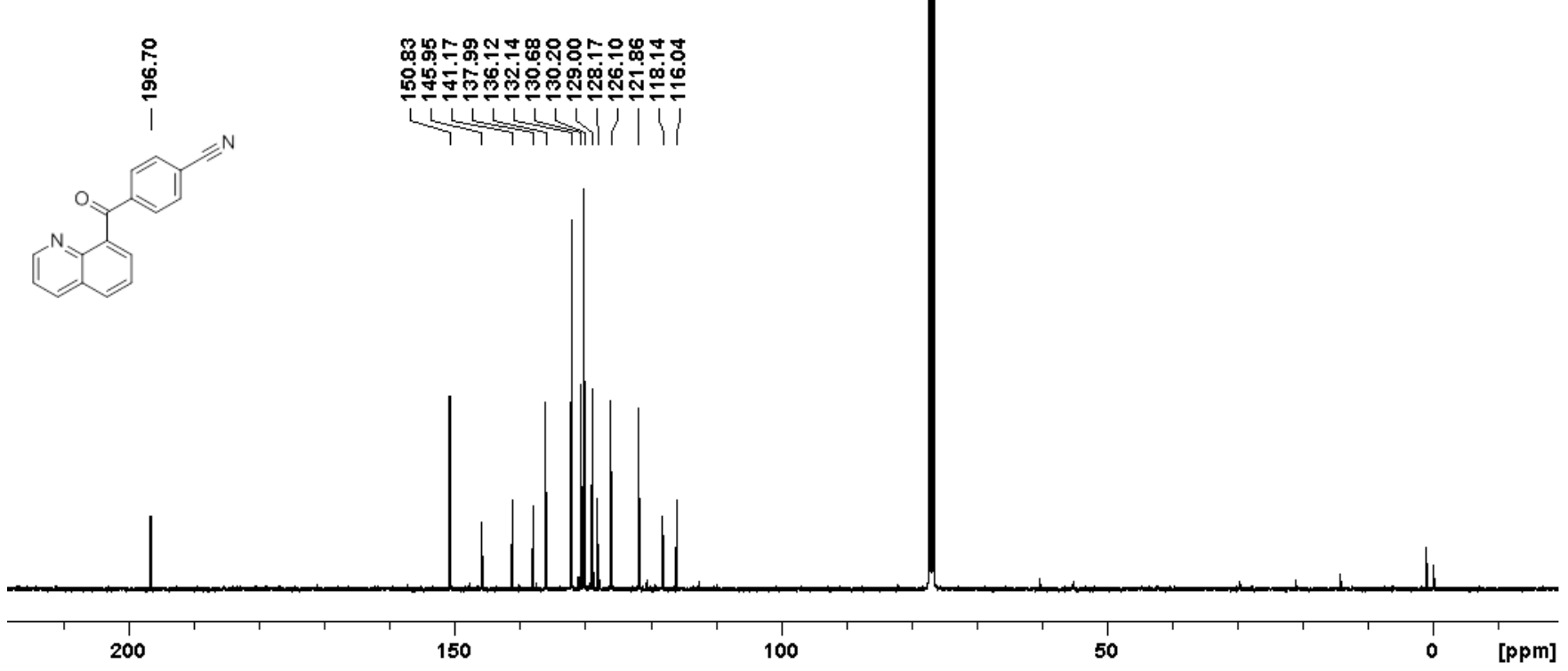


${ }^{1} \mathrm{H}$ NMR spectrum for 7

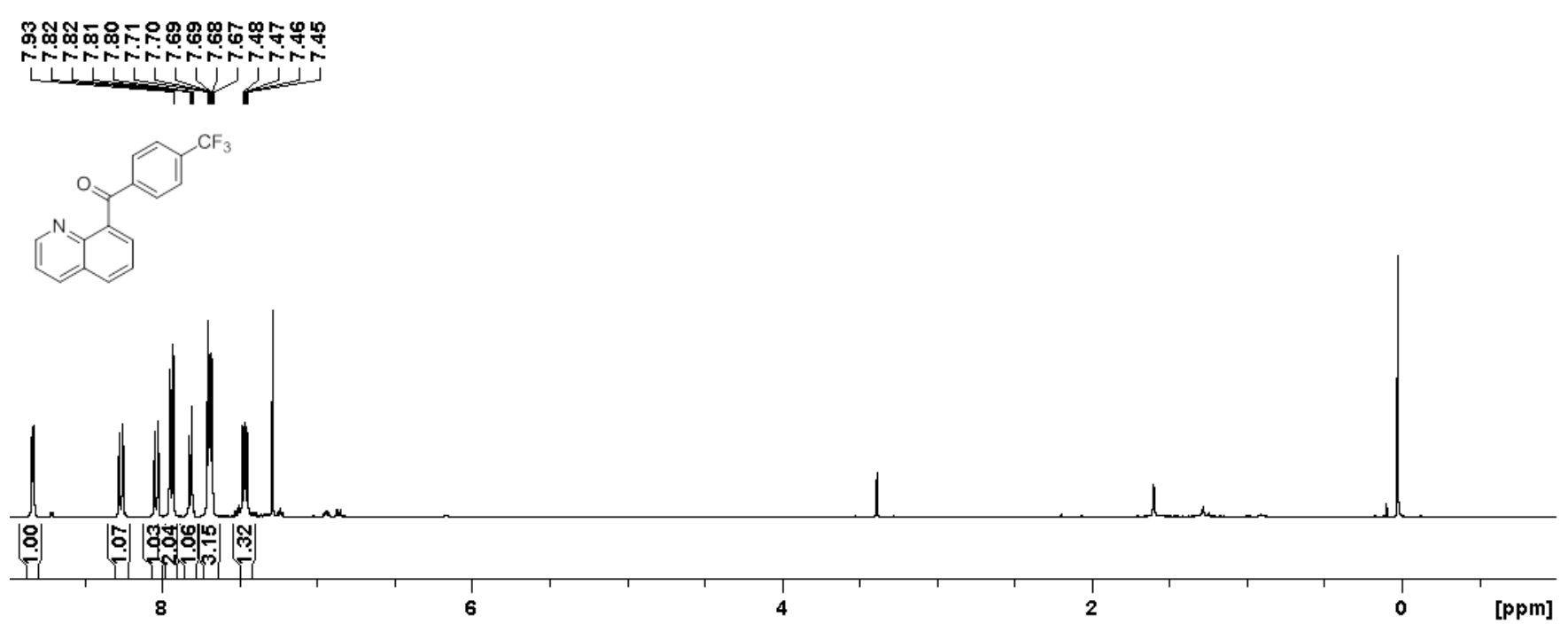

${ }^{13} \mathrm{C}$ NMR spectrum for 7

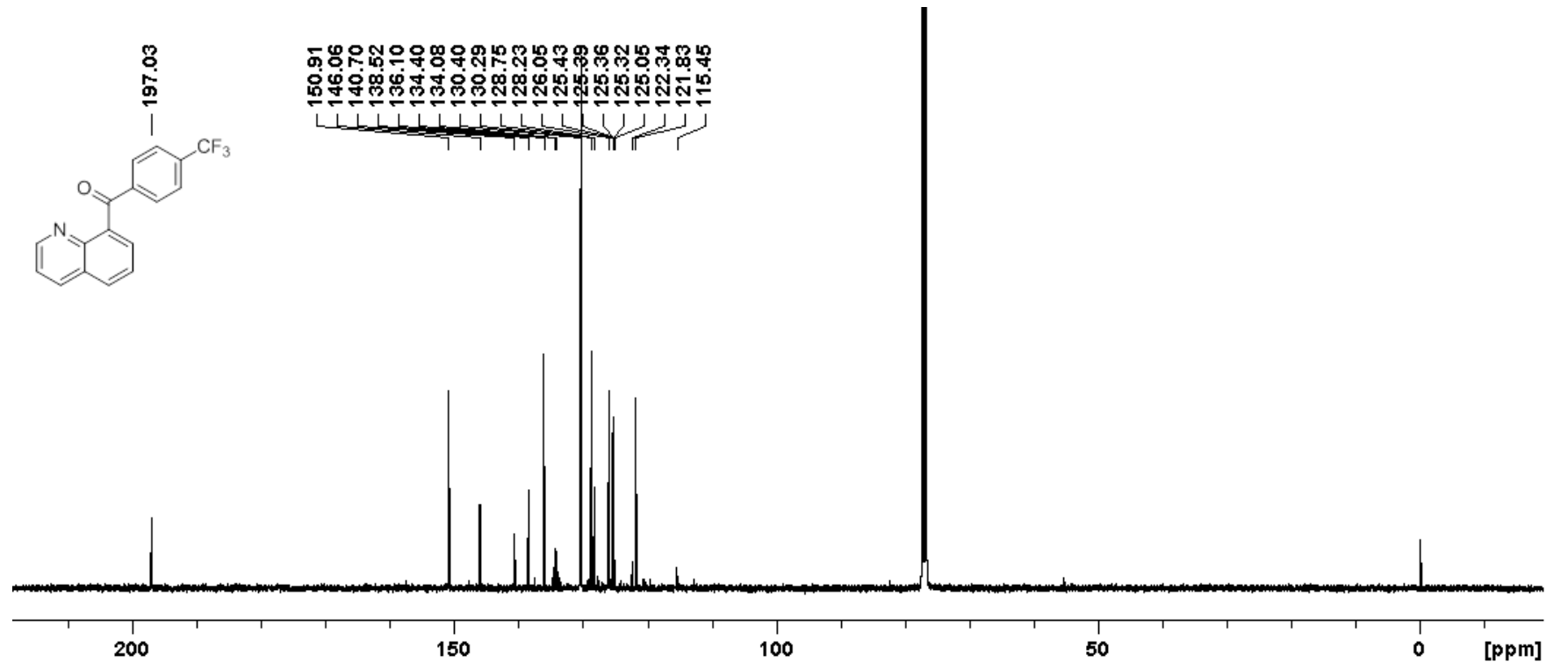


${ }^{1} \mathrm{H}$ NMR spectrum for 8

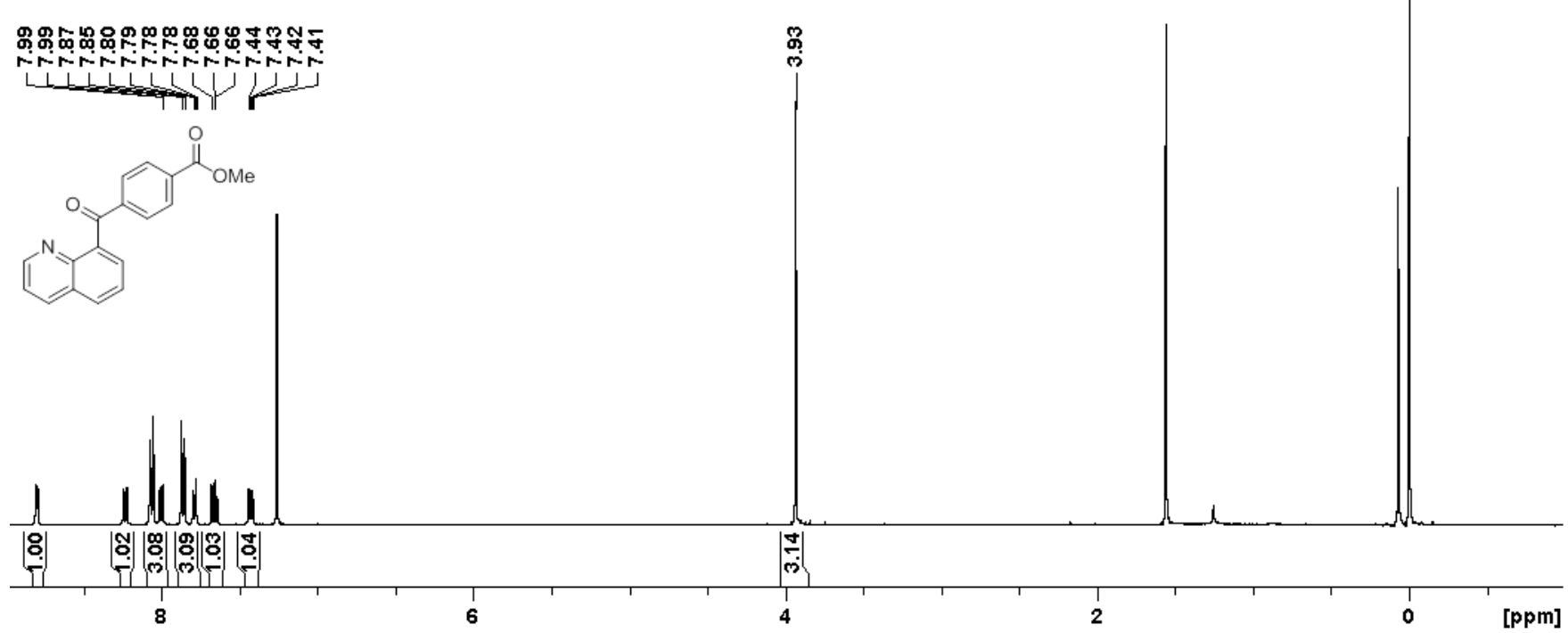

${ }^{13} \mathrm{C}$ NMR spectrum for 8

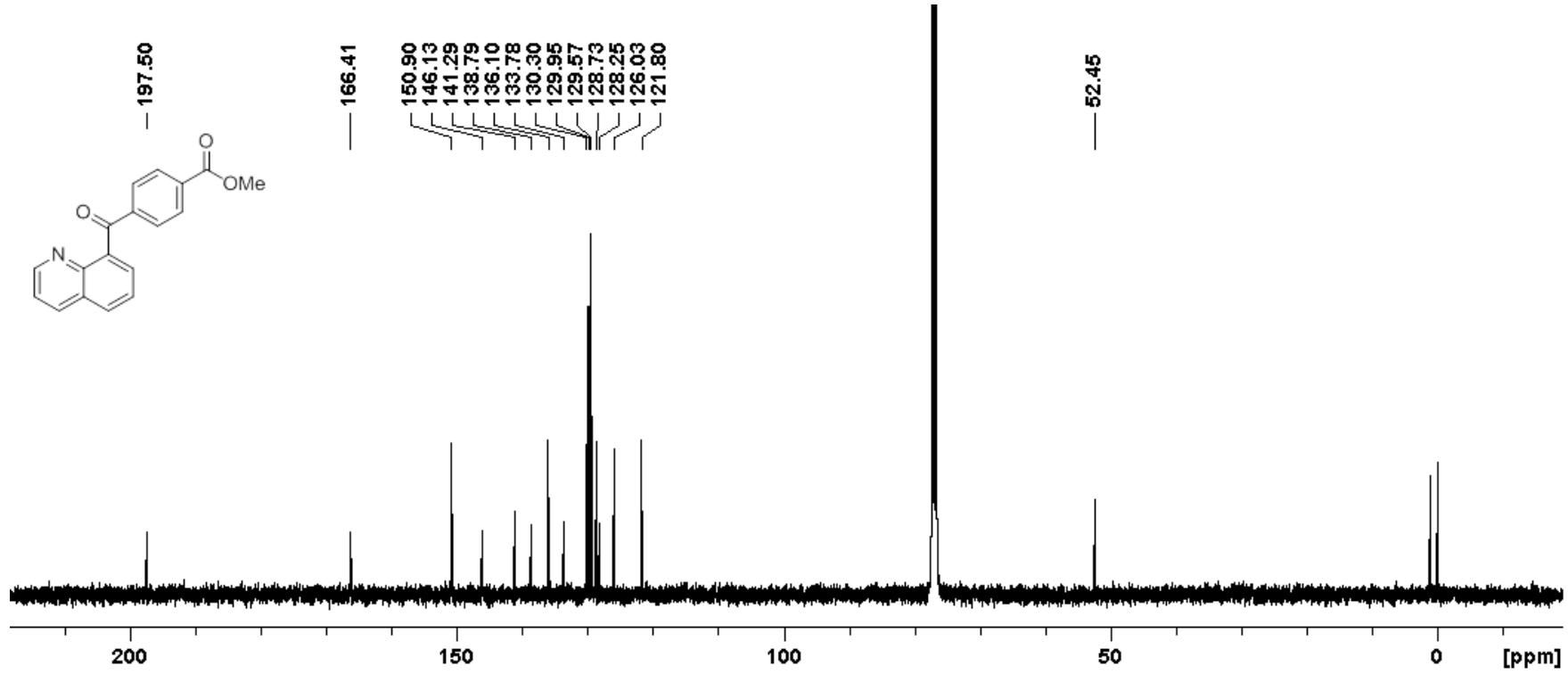


${ }^{1} \mathrm{H}$ NMR spectrum for 9

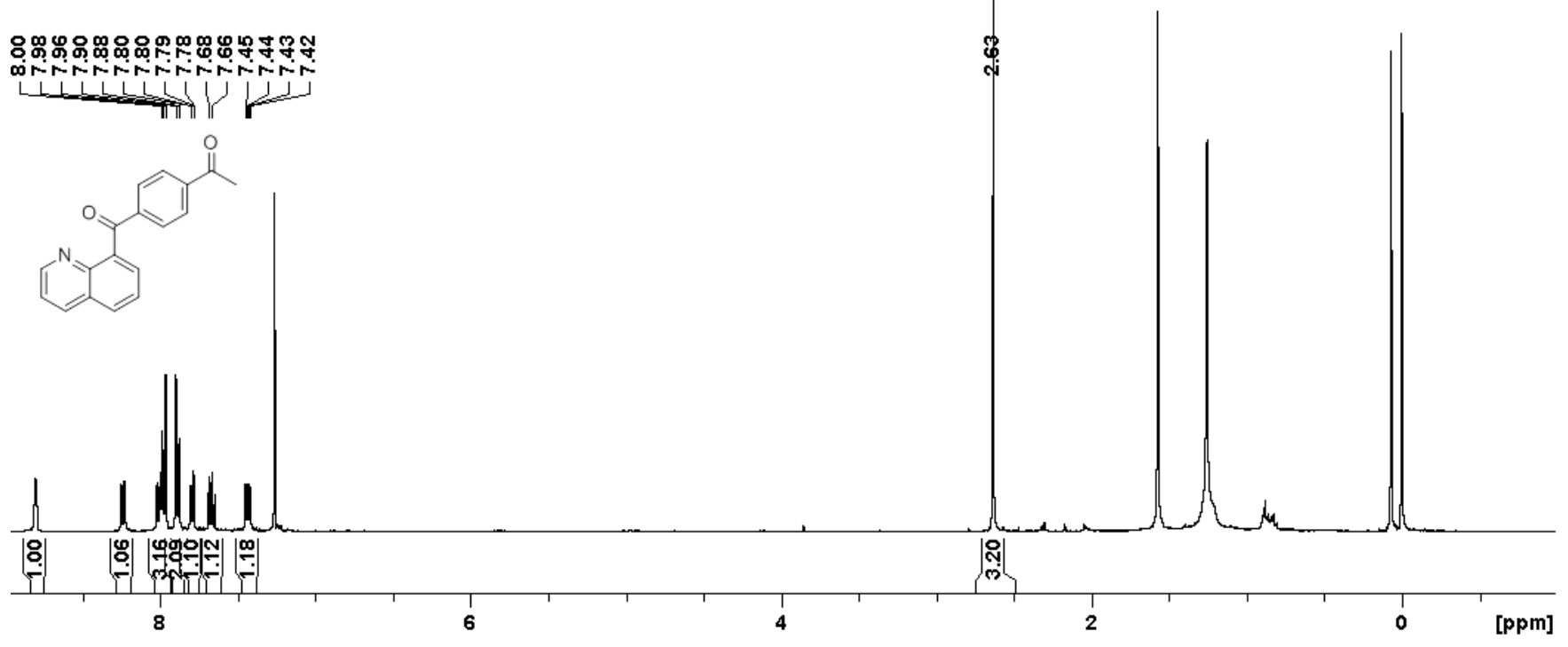

${ }^{13} \mathrm{C}$ NMR spectrum for 9

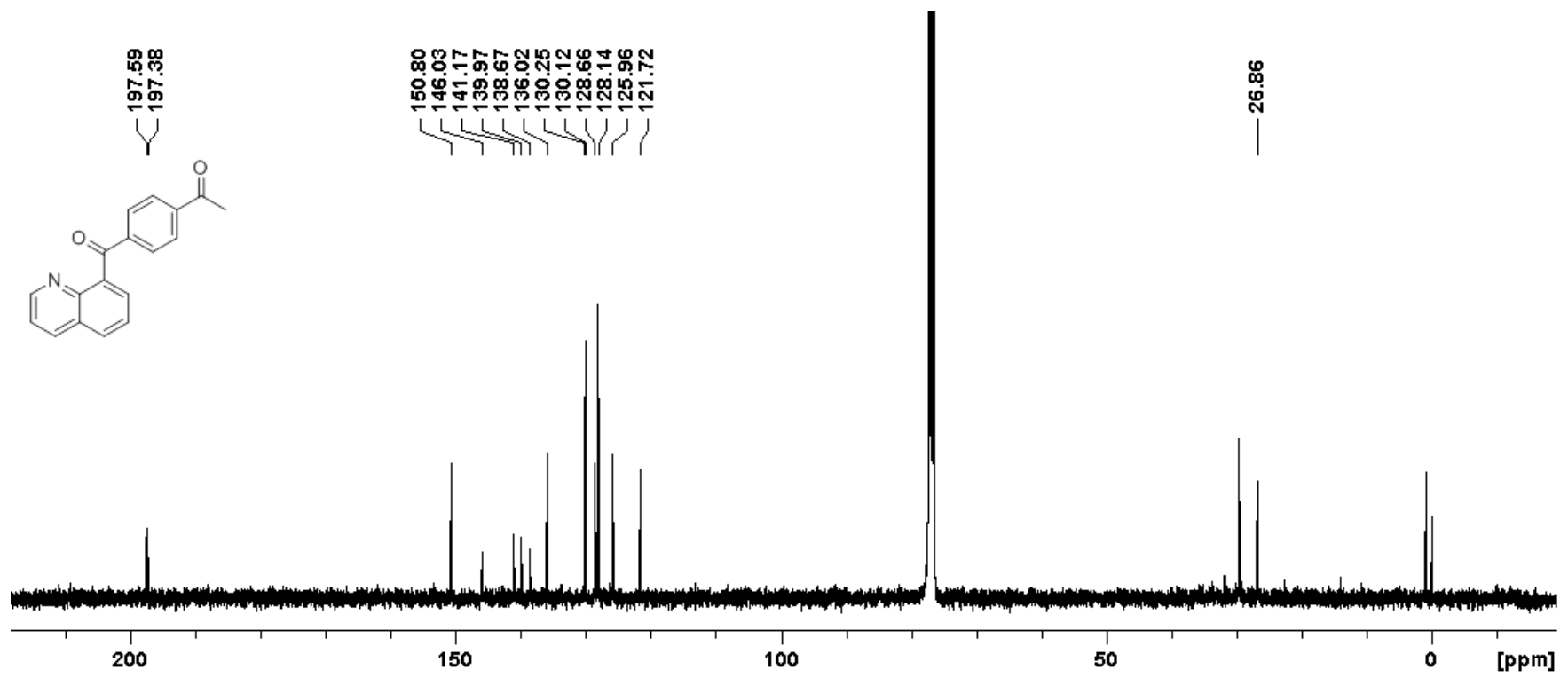


${ }^{1} \mathrm{H}$ NMR spectrum for $\mathbf{1 0}$

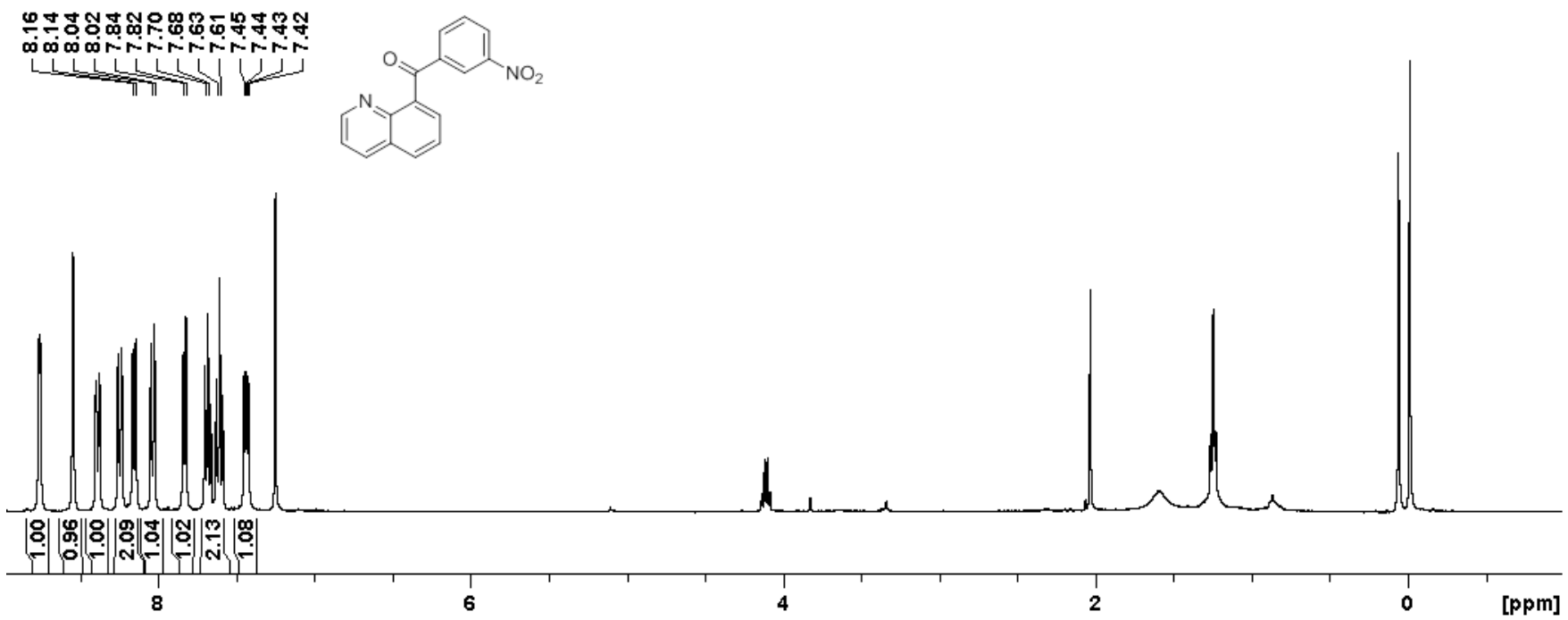

${ }^{13} \mathrm{C}$ NMR spectrum for $\mathbf{1 0}$

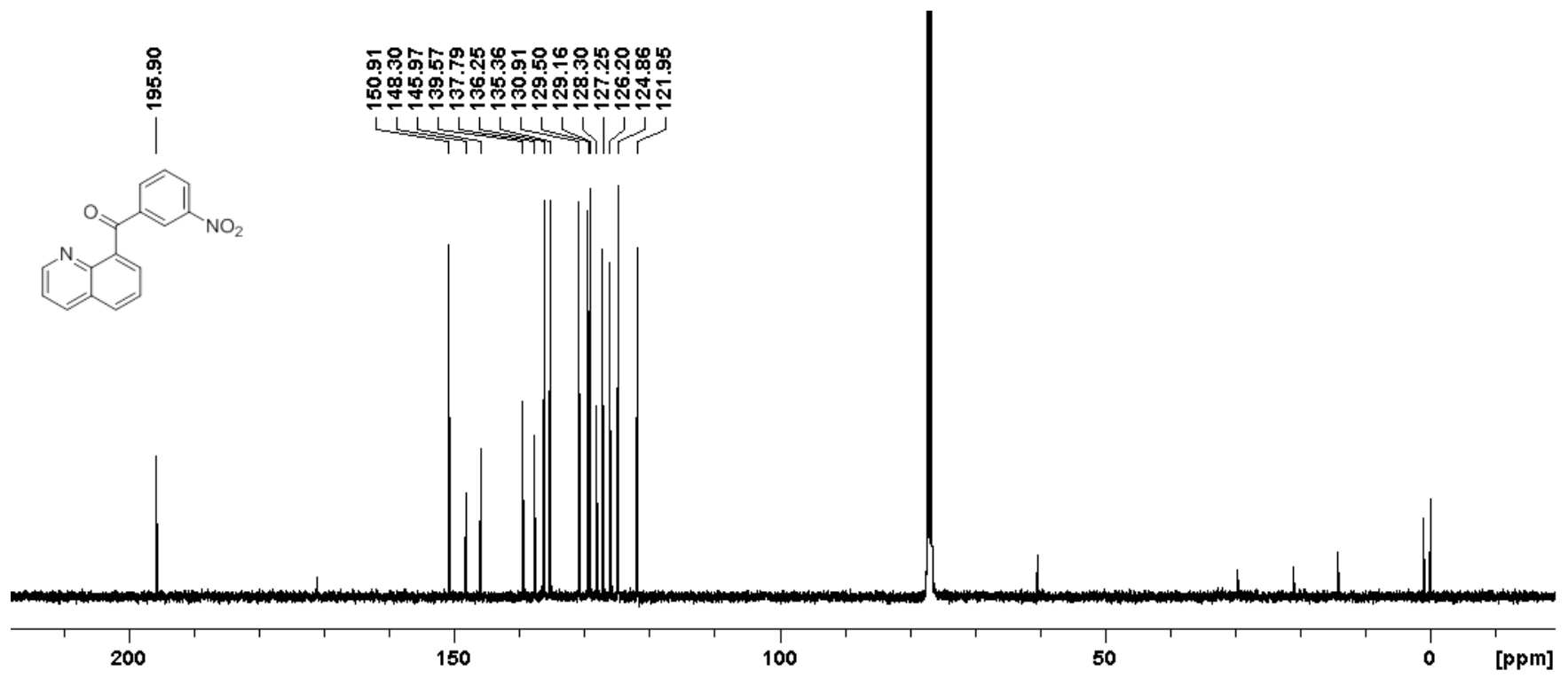


${ }^{1} \mathrm{H}$ NMR spectrum for $\mathbf{1 1}$

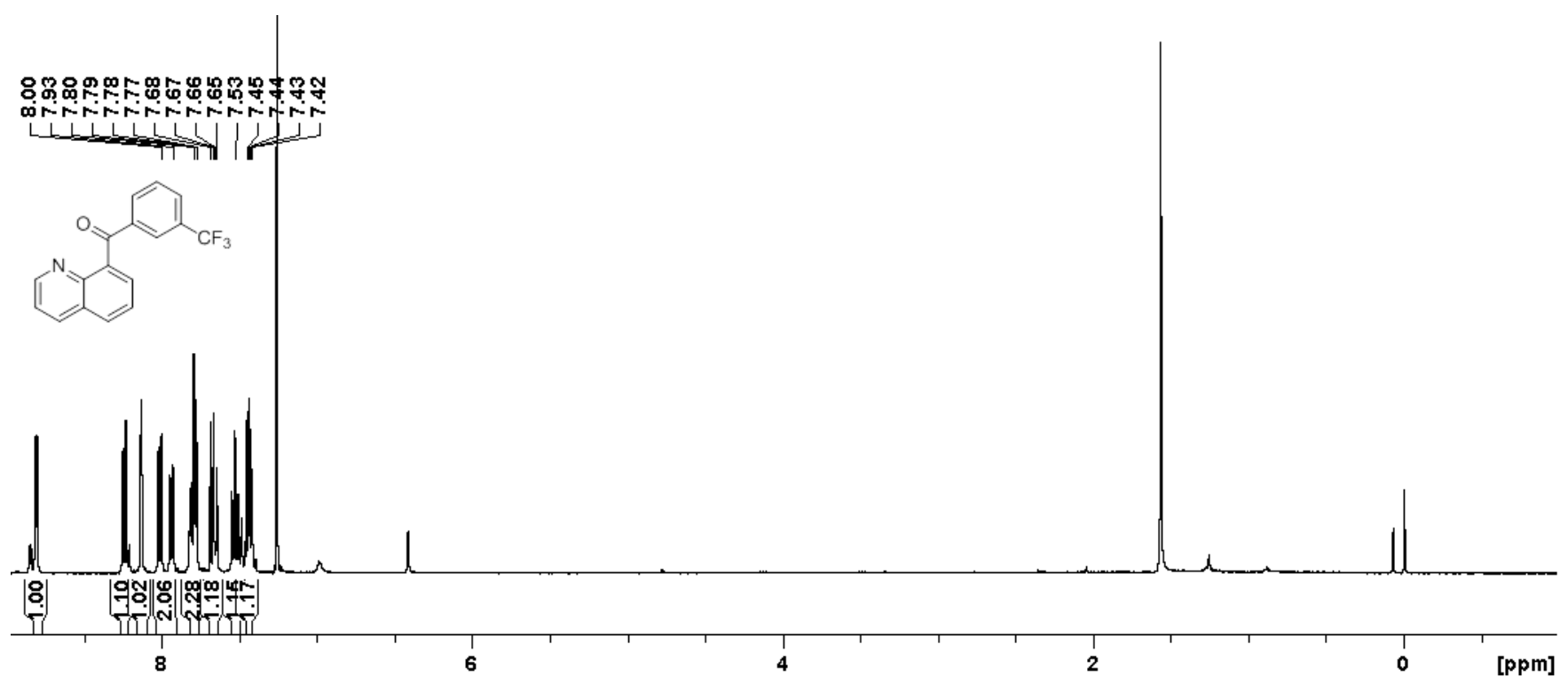

${ }^{13} \mathrm{C}$ NMR spectrum for $\mathbf{1 1}$

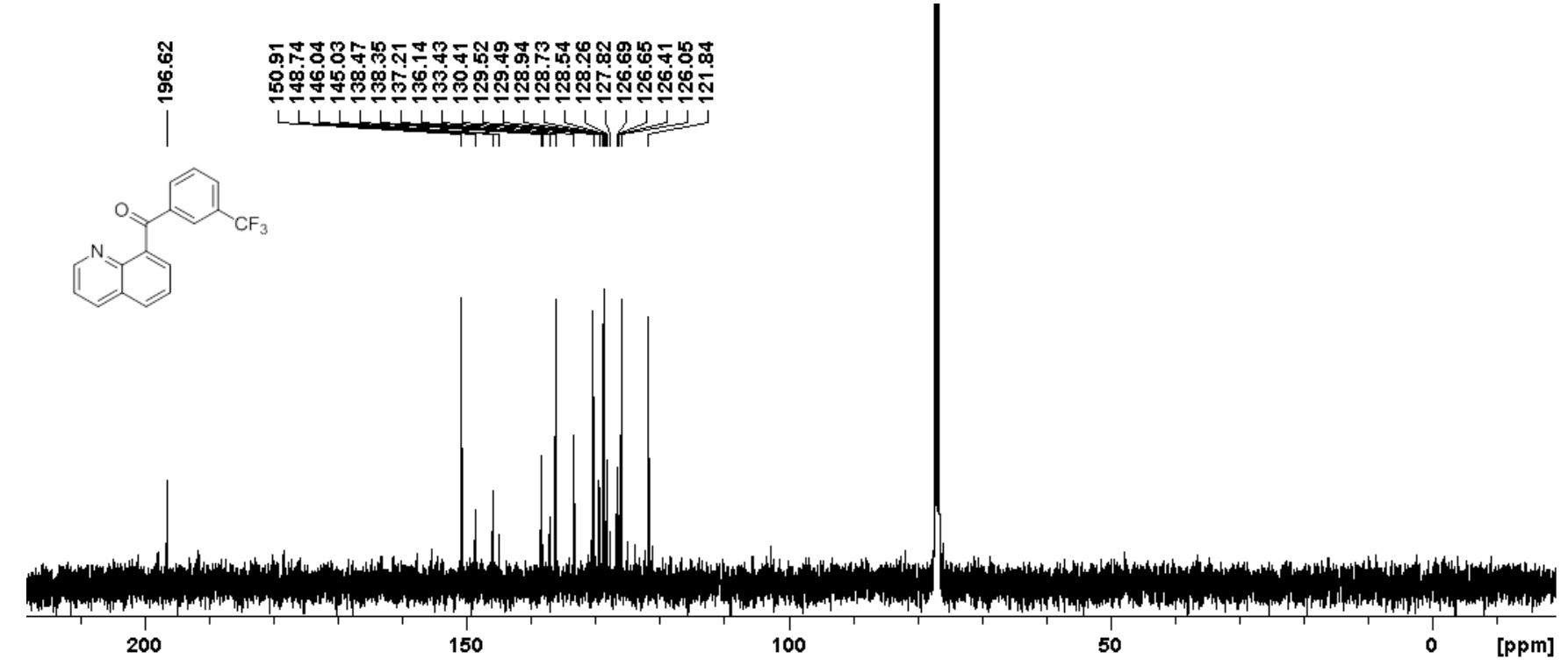


${ }^{1} \mathrm{H}$ NMR spectrum for $\mathbf{1 2}$

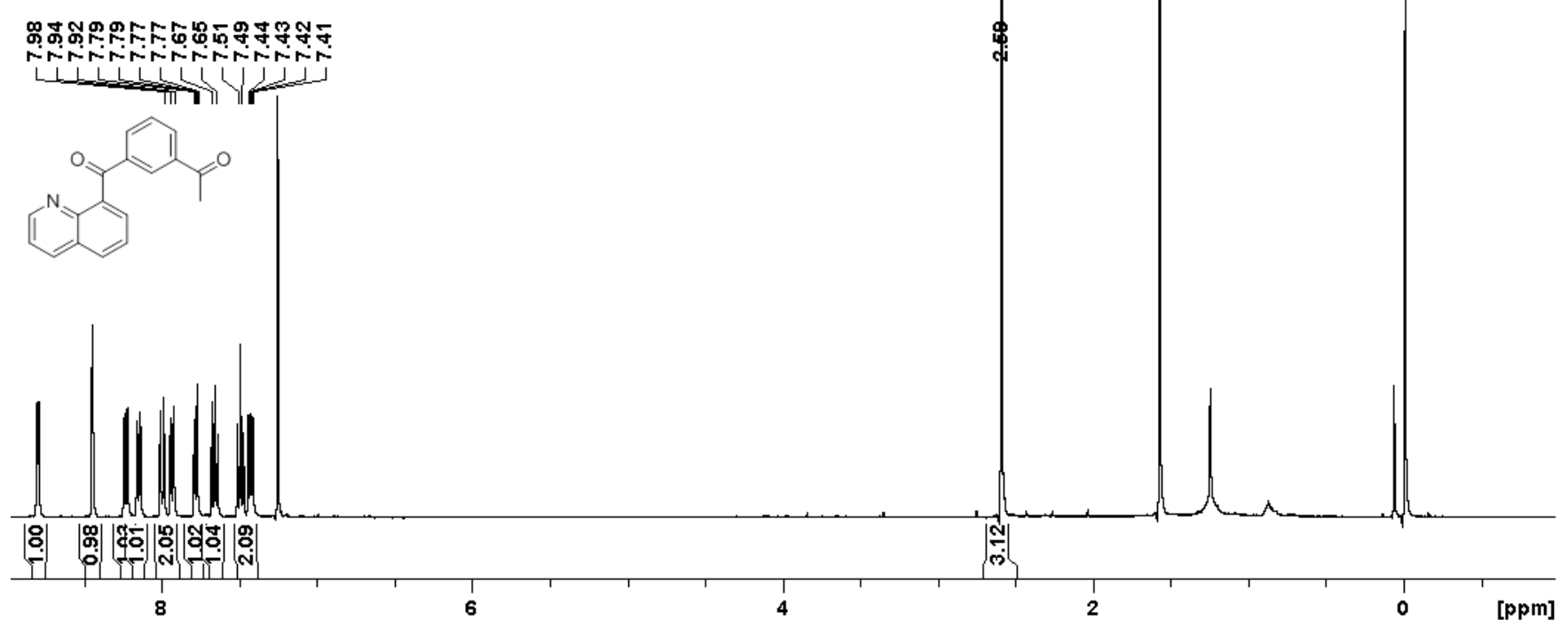

${ }^{13} \mathrm{C}$ NMR spectrum for 12

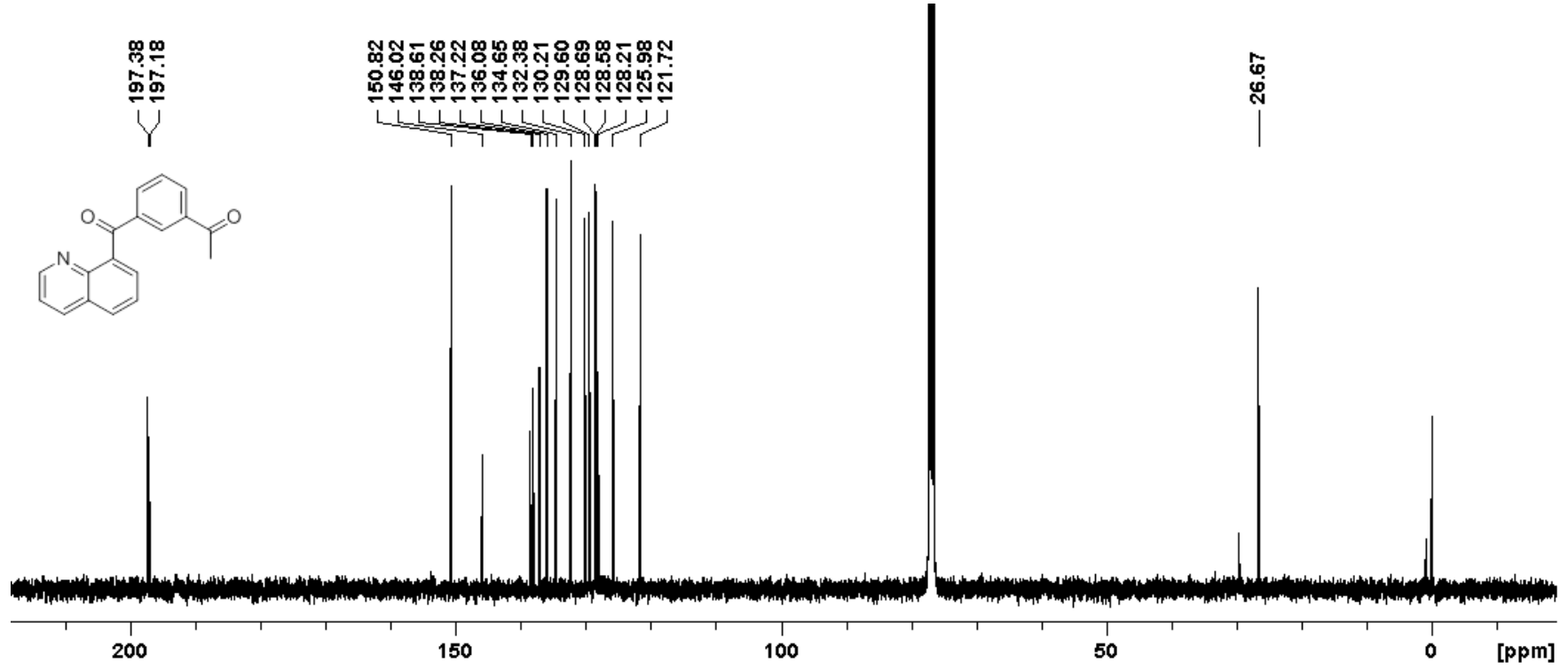


${ }^{1} \mathrm{H}$ NMR spectrum for $\mathbf{1 3}$

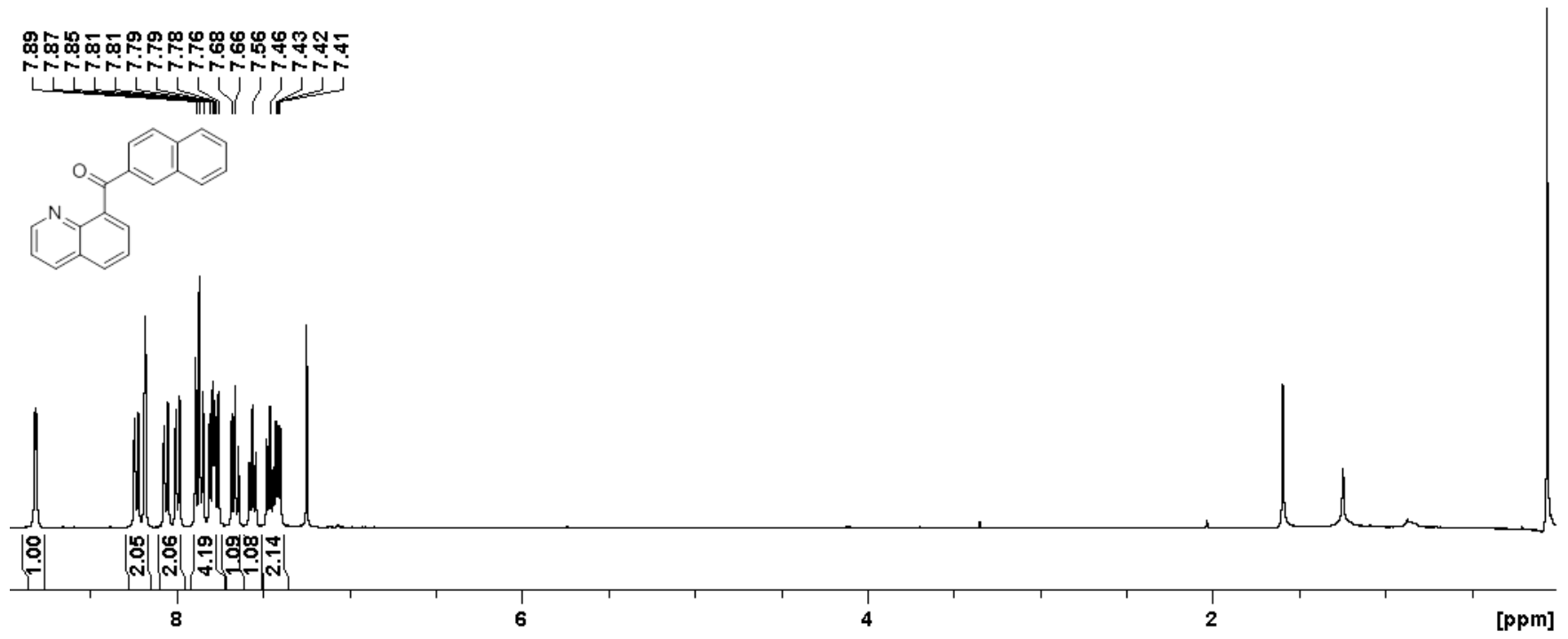

${ }^{13} \mathrm{C}$ NMR spectrum for $\mathbf{1 3}$

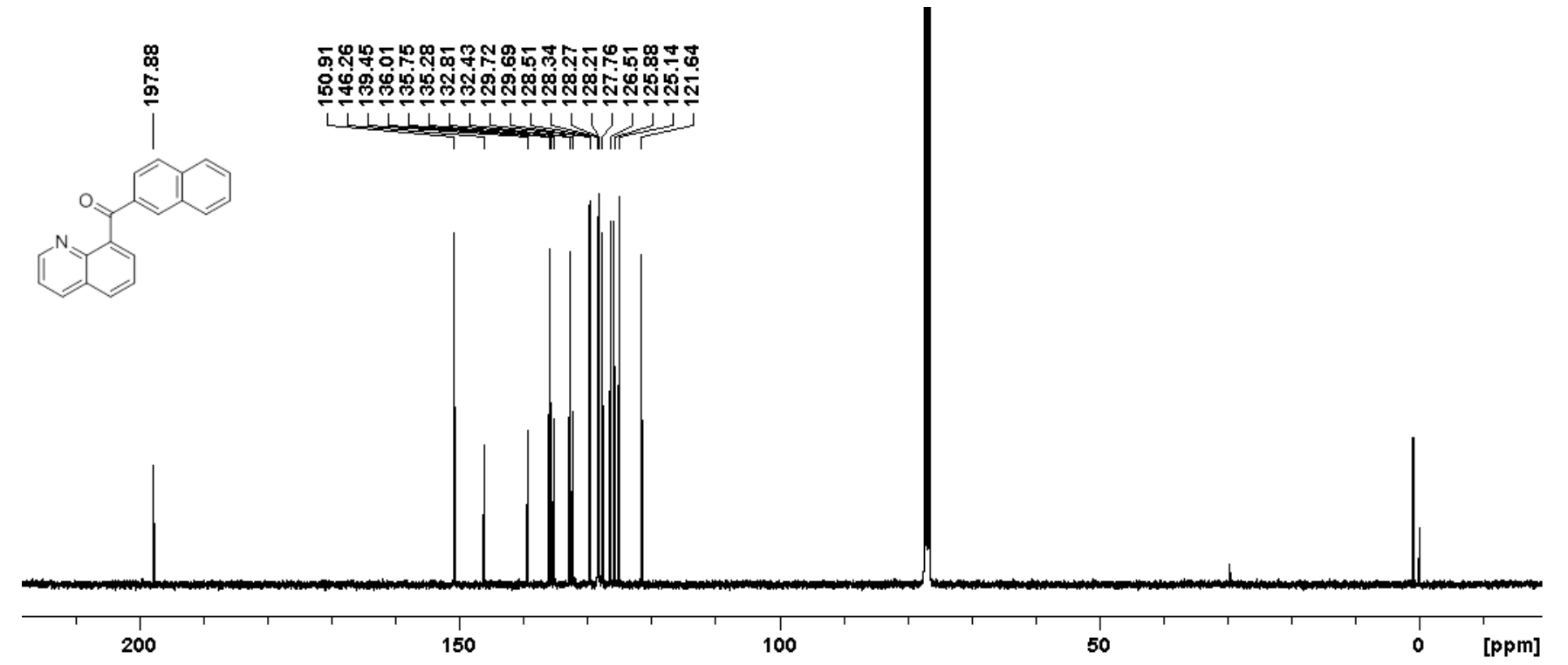


${ }^{1} \mathrm{H}$ NMR spectrum for 15

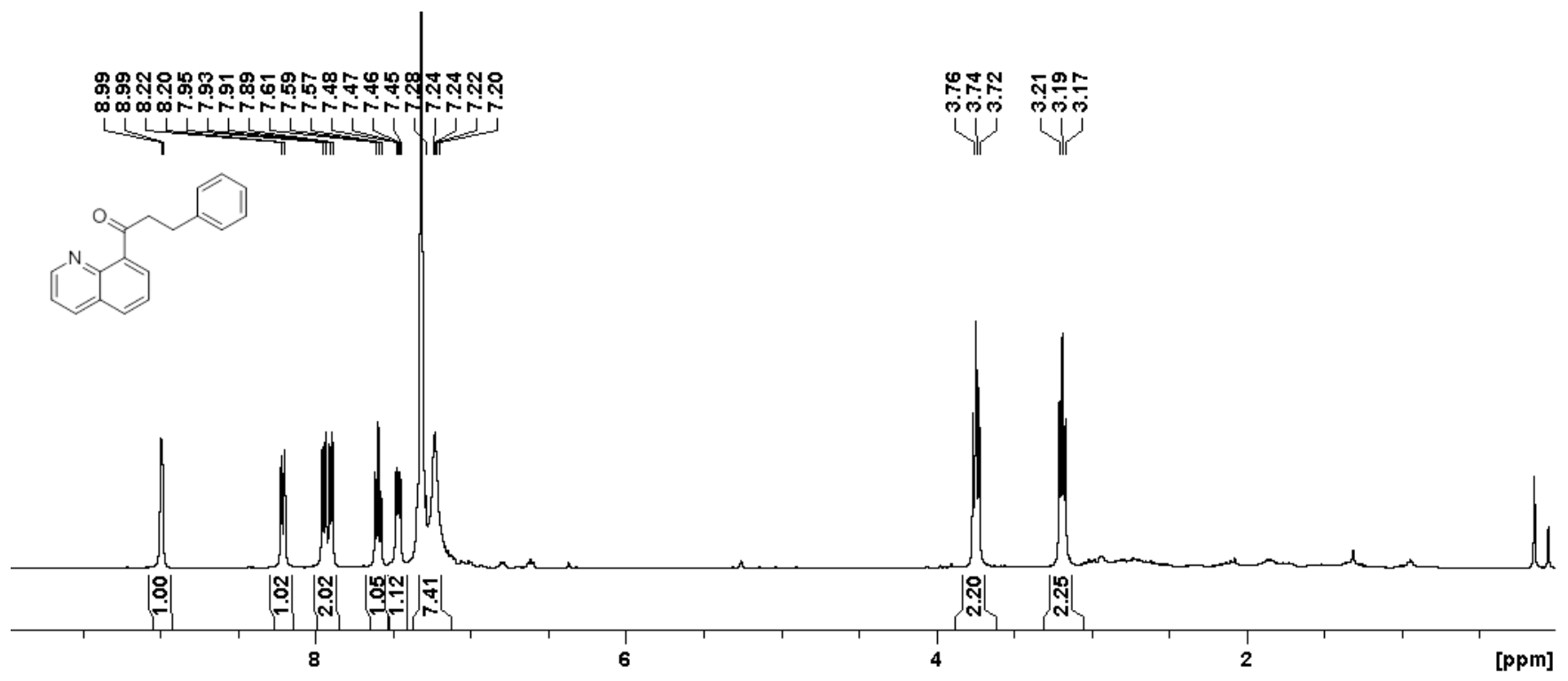

${ }^{13} \mathrm{C}$ NMR spectrum for 15

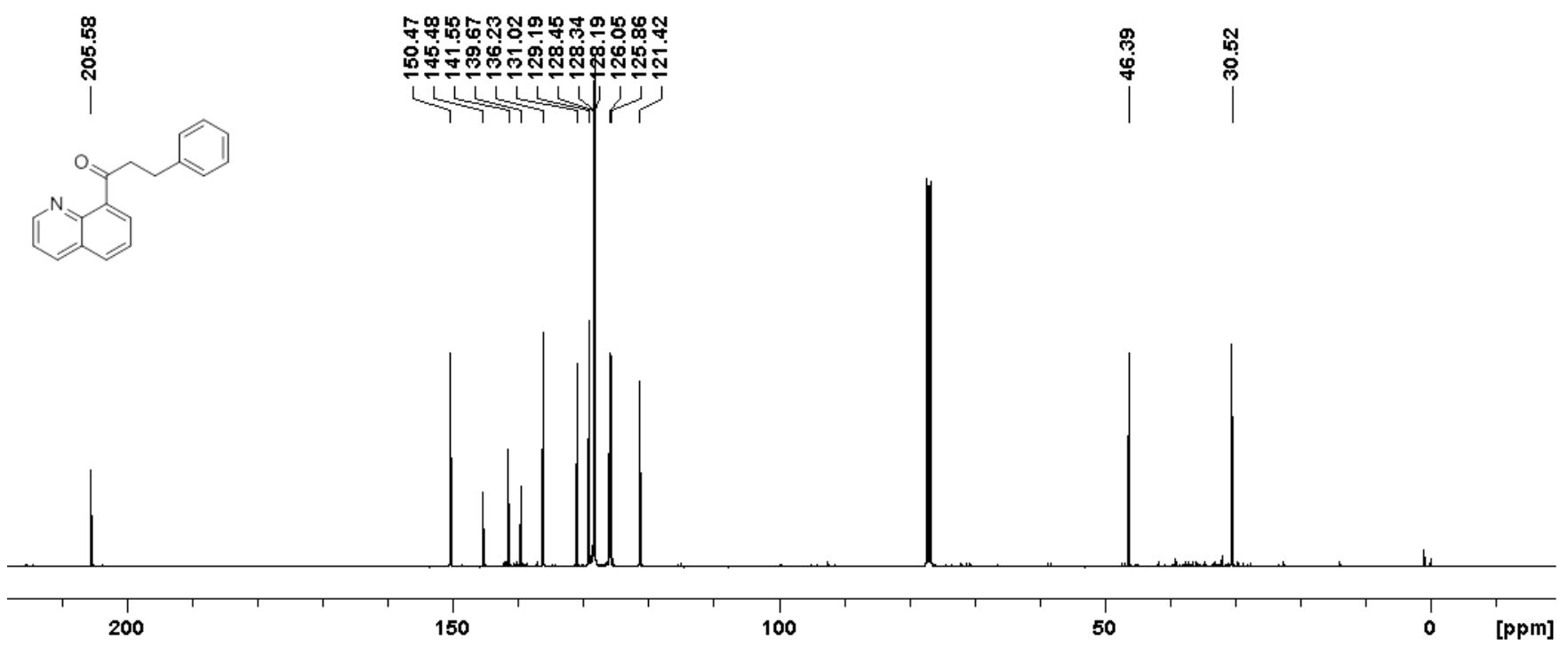


${ }^{1} \mathrm{H}$ NMR spectrum for 16

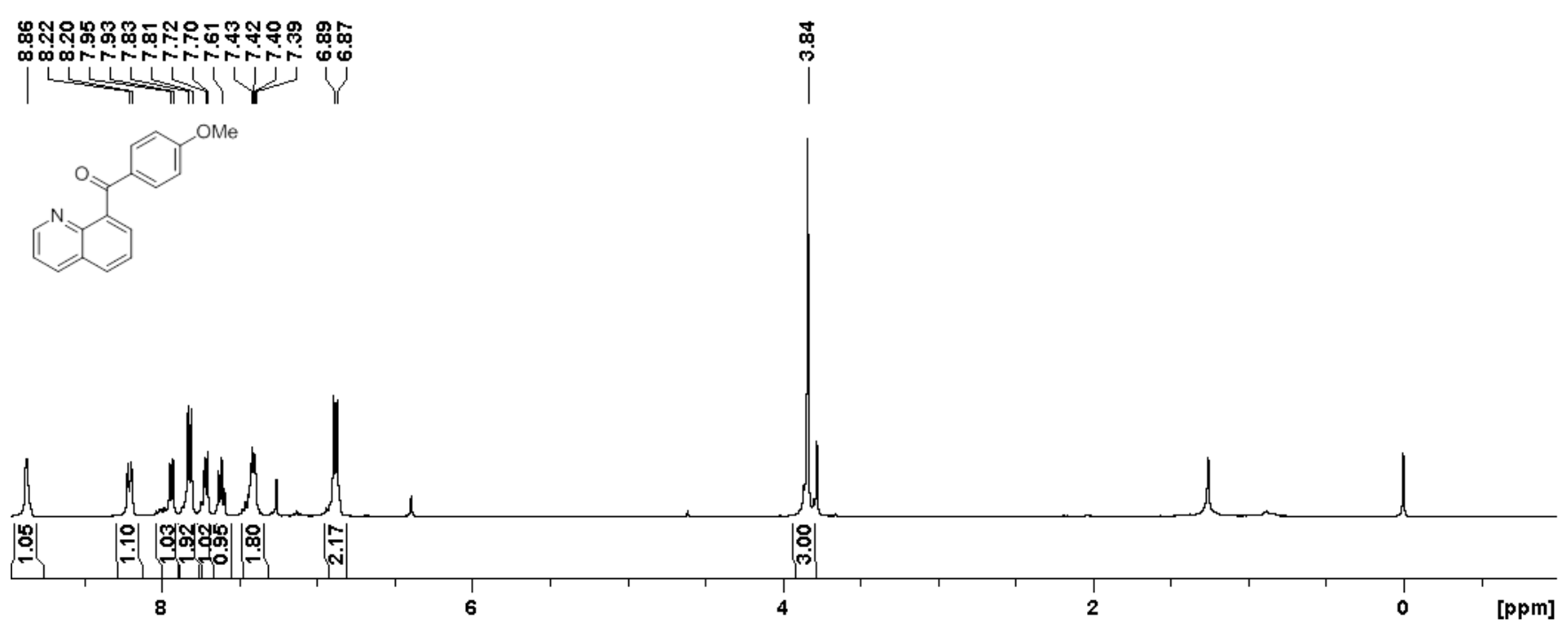

${ }^{13} \mathrm{C}$ NMR spectrum for 16

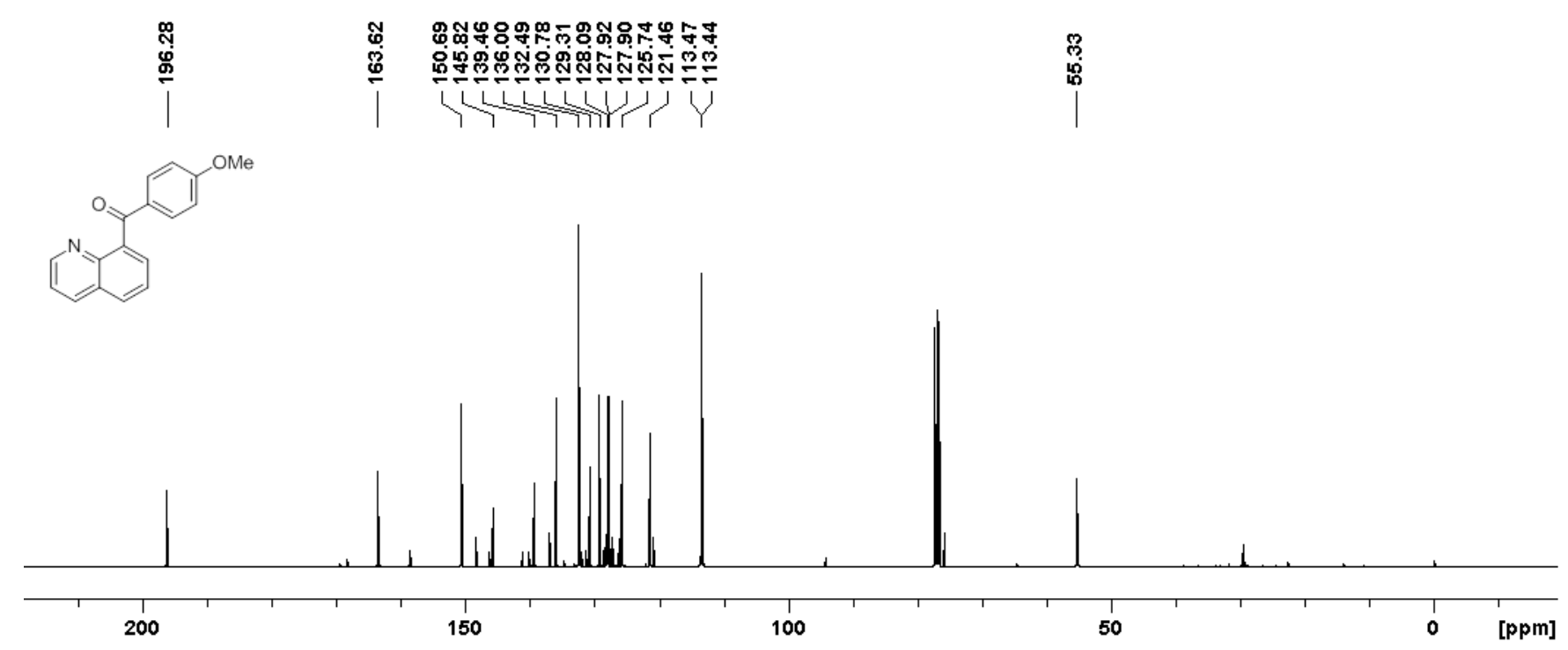


${ }^{1} \mathrm{H}$ NMR spectrum for $\mathbf{1 8}$

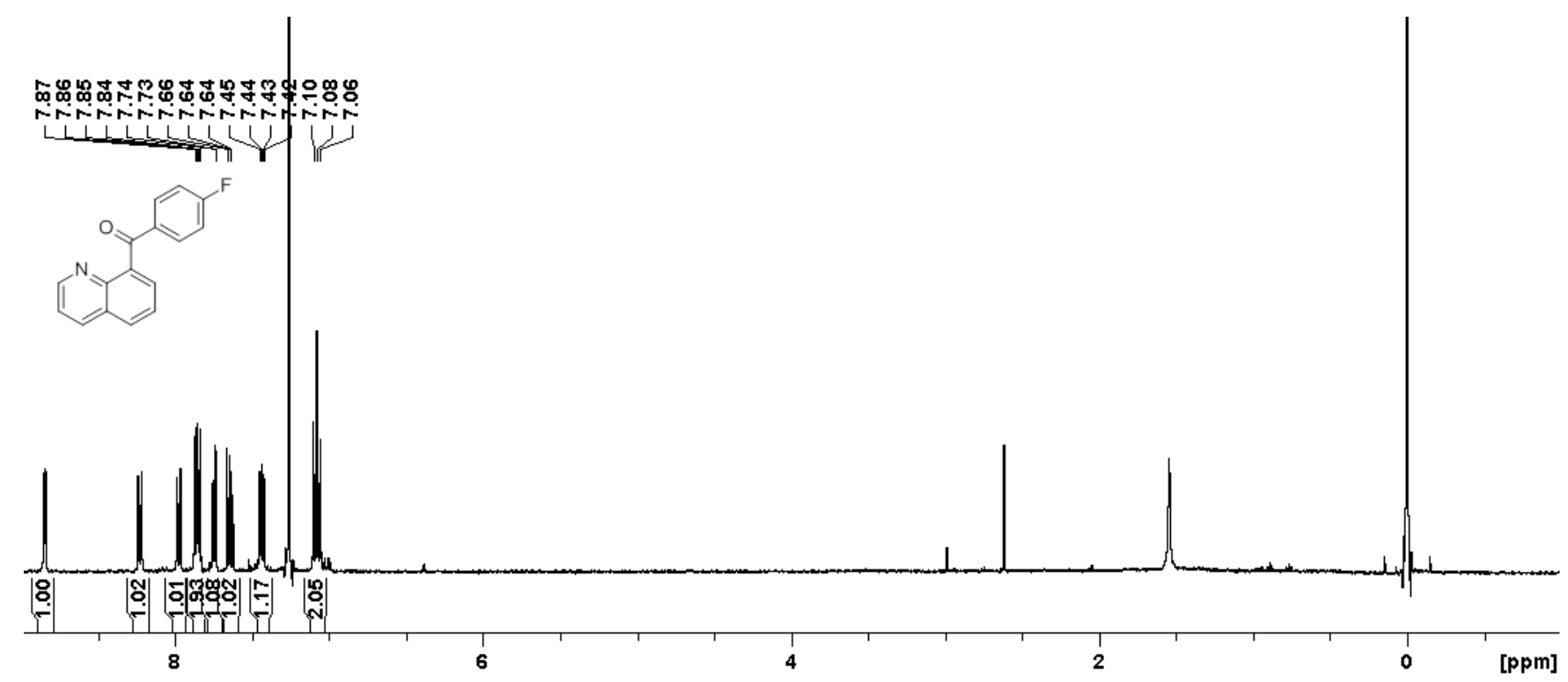

${ }^{13} \mathrm{C}$ NMR spectrum for $\mathbf{1 8}$

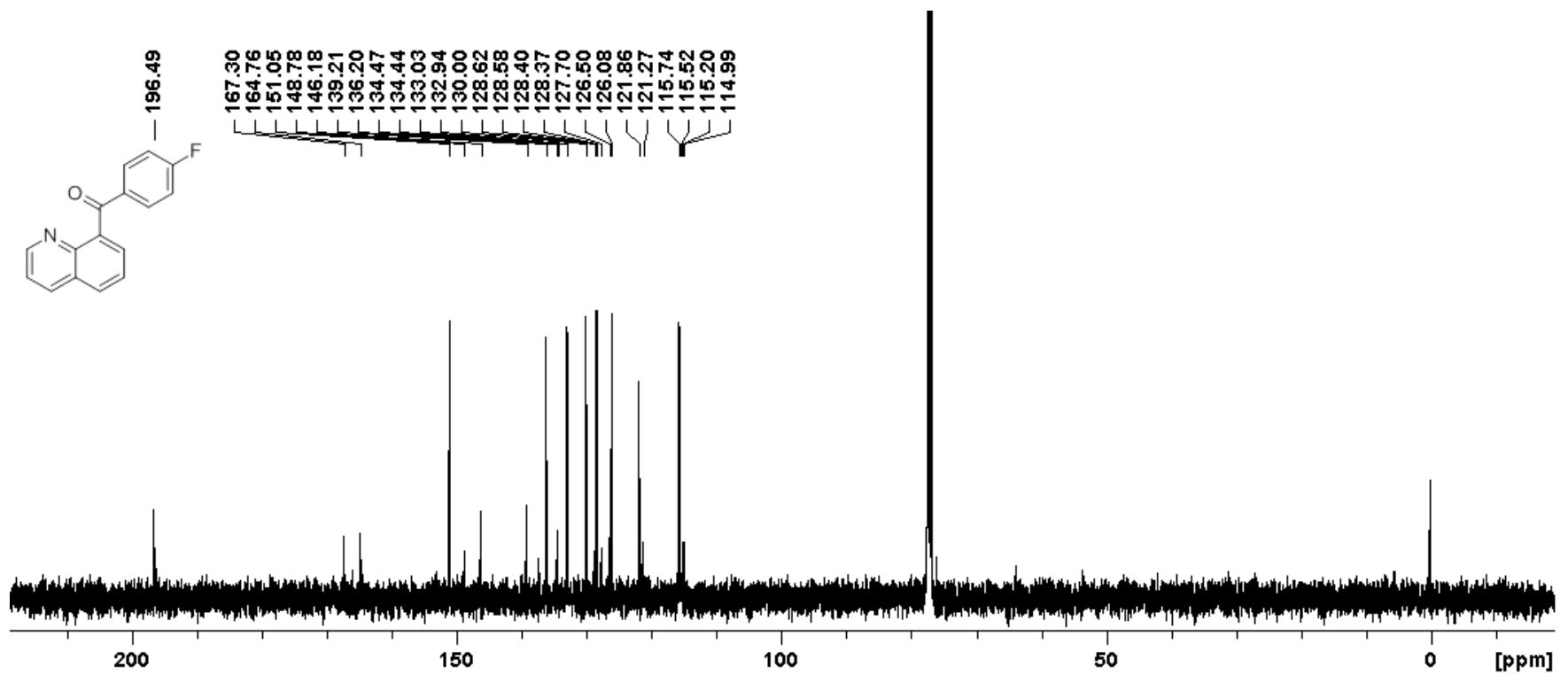


${ }^{1} \mathrm{H}$ NMR spectrum for $\mathbf{1 9}$

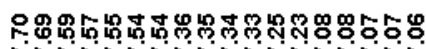

ता का
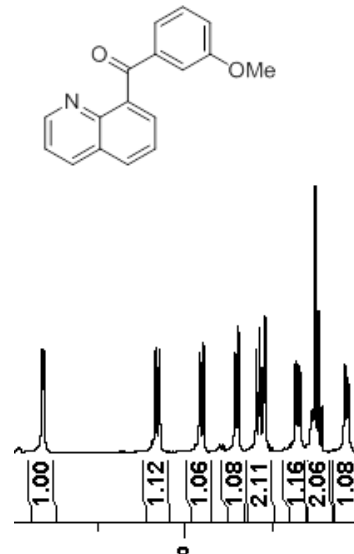

8
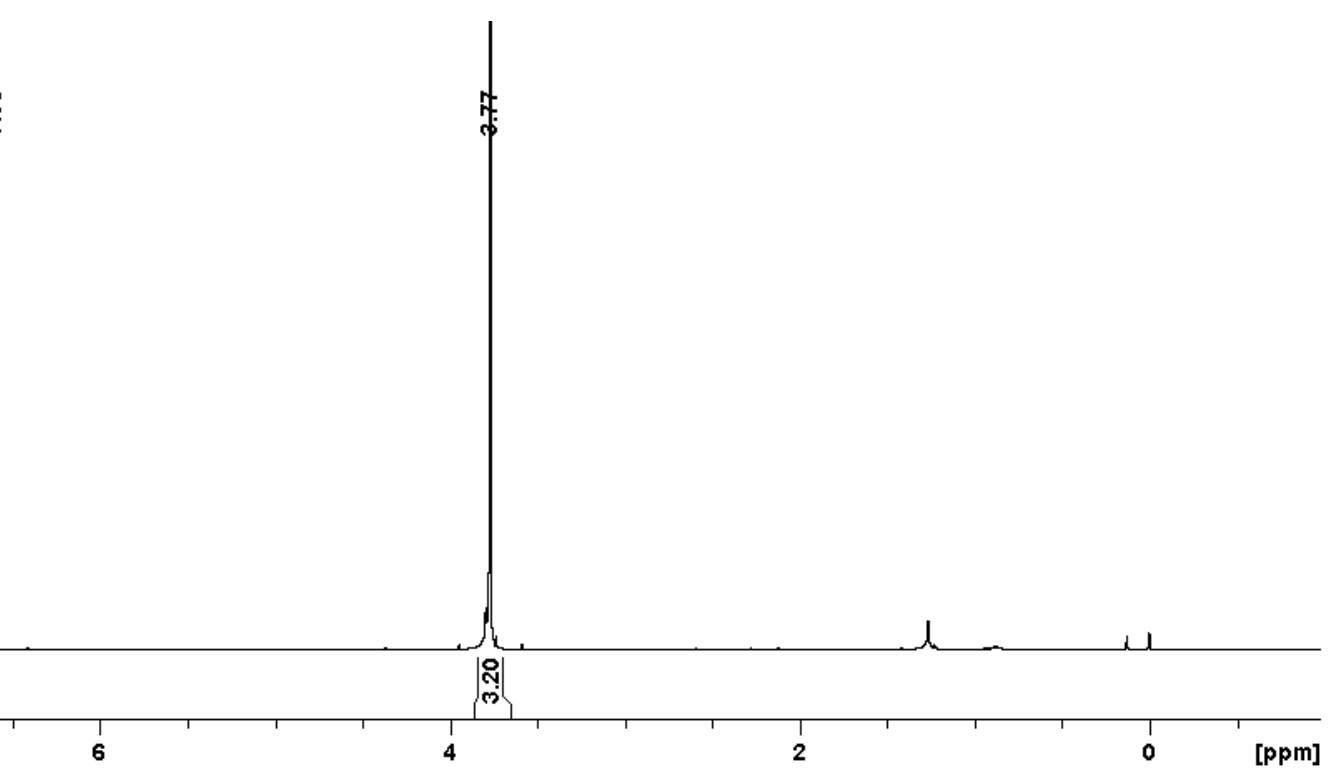

${ }^{13} \mathrm{C}$ NMR spectrum for $\mathbf{1 9}$

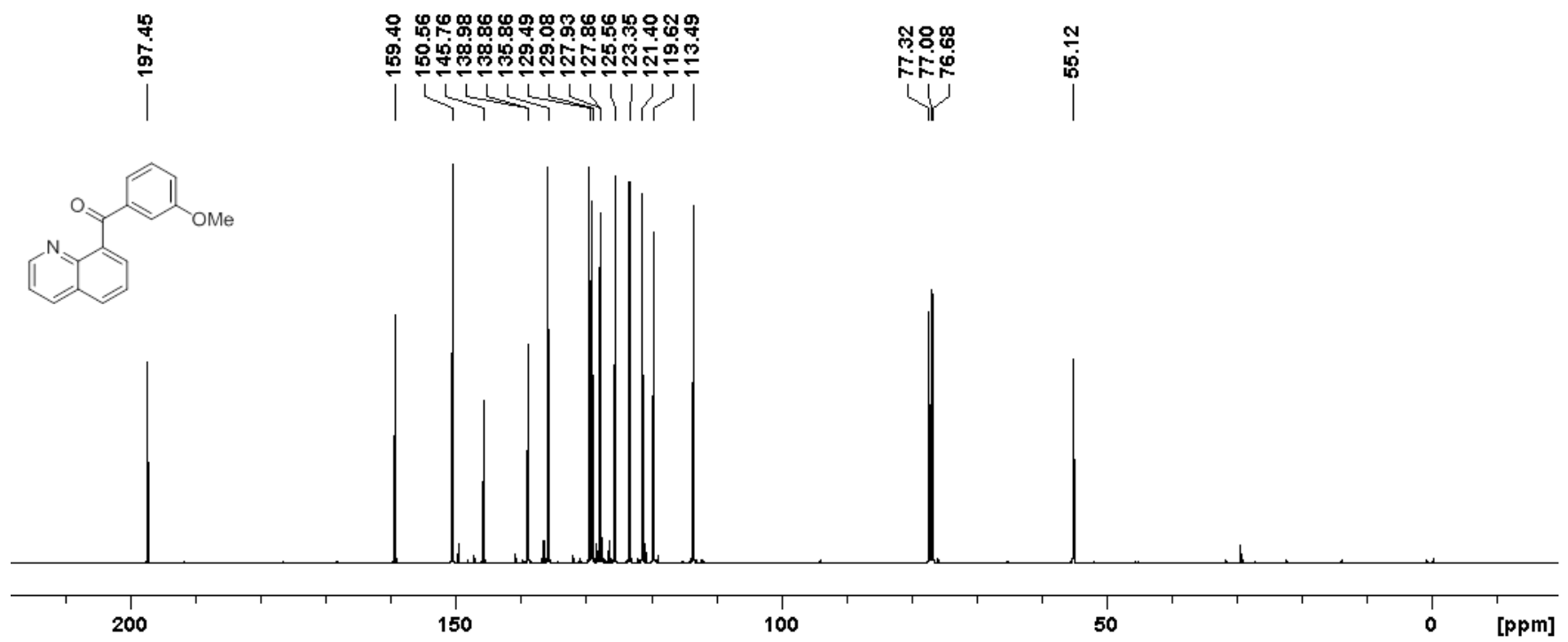




\section{References}

1) Dreis, A. M.; Douglas, C. J. J. Am. Chem. Soc. 2009, 131, 412.

2) Wentzel, M. T.; Reddy, V. J.; Hyster, T. K.; Douglas, C. J. Angew. Chem. Int. Ed. 2009, 48, 6121.

3) Wang, J.; Chen, W.; Zuo, S.; Liu, L.; Shang, Z.; Wang, J. Angew. Chem. Int. Ed. 2012, 51, 12334. 